\title{
Systematic study of multi-quasiparticle $K$-isomeric bands in tungsten isotopes by the extended projected shell model
}

\author{
Xin-Yi Wu, ${ }^{1,2}$ S. K. Ghorui, ${ }^{1,2}$ Long-Jun Wang, ${ }^{1, *}$ Yang Sun, ${ }^{1,2,3, \dagger}$ Mike Guidry, ${ }^{4}$ and Philip M. Walker ${ }^{5}$ \\ ${ }^{1}$ School of Physics and Astronomy, Shanghai Jiao Tong University, Shanghai 200240, China \\ ${ }^{2}$ Collaborative Innovation Center of IFSA, Shanghai Jiao Tong University, Shanghai 200240, China \\ ${ }^{3}$ Institute of Modern Physics, Chinese Academy of Sciences, Lanzhou 730000, China \\ ${ }^{4}$ Department of Physics and Astronomy, University of Tennessee, Knoxville, Tennessee 37996, USA \\ ${ }^{5}$ Department of Physics, University of Surrey, Guildford GU2 7XH, United Kingdom \\ (Received 24 March 2017; revised manuscript received 27 April 2017; published 16 June 2017)
}

\begin{abstract}
Background: The interplay between collective and single-particle degrees of freedom is an important structure aspect to study. The nuclei in the $A \approx 180$ mass region are often denoted as good examples to study such problems because these nuclei are known to exhibit many rotational bands based on multi-quasiparticle $K$ isomers.

Purpose: A large set of high-quality experimental data on high- $K$ isomeric states in the $A \approx 180$ mass region has accumulated. A systematic description of them is a theoretical challenge as it requires a method going beyond the usual mean field with multi-quasiparticle configurations built in the shell-model basis. The $K$-isomer data provide an ideal testing ground for theoretical models.

Method: The recently extended projected shell model (PSM) by the Pfaffian method is employed with a sufficiently large configuration space including up to 10 quasiparticles. The restoration of rotational symmetry which is broken in the deformed mean field is obtained by means of angular-momentum projection. With axial symmetry in the basis deformation, each multi-quasiparticle state, classified by a $K$ quantum number, represents the major component of a rotational $K$ band. Shell-model diagonalization in such a projected basis defines the $K$ mixing, which is the key ingredient of the present method.

Results: Quasiparticle structure and rotational properties of high- $K$ isomers in even-even neutron-rich ${ }^{174-186} \mathrm{~W}$ isotopes are described. The rotational evolution of the yrast and near-yrast bands is discussed with successive band crossings. Multi-quasiparticle $K$ isomers and associated rotational bands in each W isotope are studied with detailed quasiparticle configurations given. Electromagnetic transition properties are also studied and the calculated $B(E 2), B(M 1)$, and $g$-factors are compared with experiment if data exist.

Conclusions: Many nuclei of the $A \approx 180$ mass region exhibit properties of an axially symmetric shape and $K$ is approximately a good quantum number. For such nuclei, the extended PSM assuming an axially symmetric basis but including $K$ mixing through diagonalization of the two-body Hamiltonian is an appropriate method to study multi-quasiparticle $K$ isomers and $K$ violations in these states. For special examples where one finds highly $K$-forbidden transitions the present model needs to be further improved.
\end{abstract}

DOI: 10.1103/PhysRevC.95.064314

\section{INTRODUCTION}

Nuclear isomers [1-3] are excited, metastable quantummechanical states of nuclei. The decay of an isomeric state to lower energy states is somehow hindered, and therefore they have longer half-lives than usual excited states. In the $A \sim 180$ mass region (e.g., some $\mathrm{Hf}$ and $\mathrm{W}$ isotopes) where the nuclei are well deformed and axially symmetric, the Fermi surfaces (both neutron and proton) are based on Nilsson orbitals with large-angular-momentum projections $(K)$ on the symmetry axis [4]. Two or more states with high $K$ quantum numbers can couple to form a high- $K$ multi-quasiparticle (multi-qp) configuration. The selection rules of electromagnetic decay hinder transitions from a high- $K$ state to normal (low- $K$ ) states, resulting in a long-lived $K$ isomer [1]. Understanding nuclear isomeric states is one of the current topics in nuclear

\footnotetext{
*Present address: Department of Physics and Astronomy, University of North Carolina, Chapel Hill, NC, 27516-3255, USA.

†sunyang@sjtu.edu.cn
}

structure physics [5-7]. The current experiments are able to find evidence for six- or eight-qp isomers (see, for example, Refs. [8,9]), and discoveries of more high- $K$ isomers are expected from modern facilities such as storage rings [10]. These data provide us with valuable information on the interplay between the single-particle states and collective nuclear motion in deformed potentials.

Another potentially important but much-less-explored research field is isomeric states in nuclear astrophysics, which are expected, for some special cases, to play key roles in the determination of element production in the universe [11,12]. The interest is to understand the structure of the excited isomers as well as the intermediate states through which the isomers can be excited or deexcited, when these states are in a thermal bath of stellar temperatures. An acute example is ${ }^{180} \mathrm{Ta}^{m}$, the only naturally occurring nuclear isomer at an excitation energy of $75 \mathrm{keV}$ but with a long mean life of $>1.2 \times 10^{15} \mathrm{y}$, which gives it a special place in nuclear-astrophysics investigations. The production and survival of ${ }^{180} \mathrm{Ta}$ in stars presents a nuclear astrophysics puzzle, and its stellar nucleosynthesis has been the subject of conjecture. It was shown that the study 
of isomer decay and the identification of the intermediate states $[13,14]$ could help in understanding the astrophysical questions.

$K$ isomers have been studied in various methods, e.g., the cranked mean-field model or the tilted axis cranking model [15-18], the Wood Saxon quasiparticle random-phase approximation model and random-phase approximation method based on the sloping surface [19], and the configuration-constrained nuclear potential energy calculations [20]. However, if one wants to describe $K$ isomers and their associated rotational bands on an equal footing, then one has to go beyond the mean field and perform shell-model-like calculations. To study heavy, deformed nuclei, a practical way to build the model space is to start from a deformed single-particle basis and perform angular-momentum projection for the deformed $\mathrm{qp}$ states. Shell-model diagonalization with a two-body Hamiltonian is then carried out in the projected multi-qp space. This is the basic concept of the projected shell model (PSM) [21], which has been extensively applied to nuclear structure studies. In practice, since the involved overlap matrix elements of multi-qp states are usually calculated with the generalized Wick's theorem [22], one may encounter a practical problem of combinatorial complexity when more than four-qp states are included in the basis configurations, for example, as many as hundreds (thousands) of terms have to be considered to express each matrix element with a four-qp (six-qp) state. This limitation has recently been relaxed [23] with the help of the Pfaffian method, which we shall briefly mention in the next section.

In this paper, we investigate the microscopic structure of even-even ${ }^{174-186} \mathrm{~W}$ isotopes using the extended projected shell model $[23,24]$, which is an extended version of the original PSM [21]. The extension in the multi-qp configurations of the PSM by including up to 10-qp states [23,24] provides a suitable framework to study high- $K$ isomers and the associated rotational bands. The $A \approx 180$ mass region is marked as a playground for well-deformed axial symmetric nuclei. In a simple picture, the ratio of the excitation energies of the first $4^{+}$and $2^{+}$states $\left(E_{4^{+}} / E_{2^{+}}\right)$can be used to distinguish between an axially symmetric deformed rotor $\left(E_{4^{+}} / E_{2^{+}}=\right.$ $3.33)$, a spherical vibrational nucleus $\left(E_{4^{+}} / E_{2^{+}}=2.0\right)$, and a triaxial rotor $\left(E_{4^{+}} / E_{2^{+}}=2.5\right)$ [25]. The experimental data [26] for $E_{4^{+}} / E_{2^{+}}$of the $\mathrm{W}$ isotopes with $N=98$ to 116 are summarized in Fig. 1. It can be seen that for the $N=116$ isotope, $E_{4^{+}} / E_{2^{+}}$approaches the triaxial limit. But for lower mass isotopes of ${ }^{174-186} \mathrm{~W}$ (with $N=100$ to 112 ), large deformation with axial symmetry is the main character.

The paper can be outlined as follows. In Sec. II, we briefly describe the theoretical formalism for the projected shell model used to obtain the nuclear many-body wave functions and other observables (e.g., energy levels, electromagnetic properties) for the considered isotopic chain. Section III contains our results. First, we discuss the quasiparticle configurations and band diagram for the nuclei. Subsequently, the energy bands including $K$ isomers of various isotopes are discussed in detail. Then the behavior of the yrast structures are analyzed with moments of inertia. Last, we investigate the electromagnetic properties of yrast and $K$-isomeric bands. Finally, in Sec. IV, we summarize the important results.

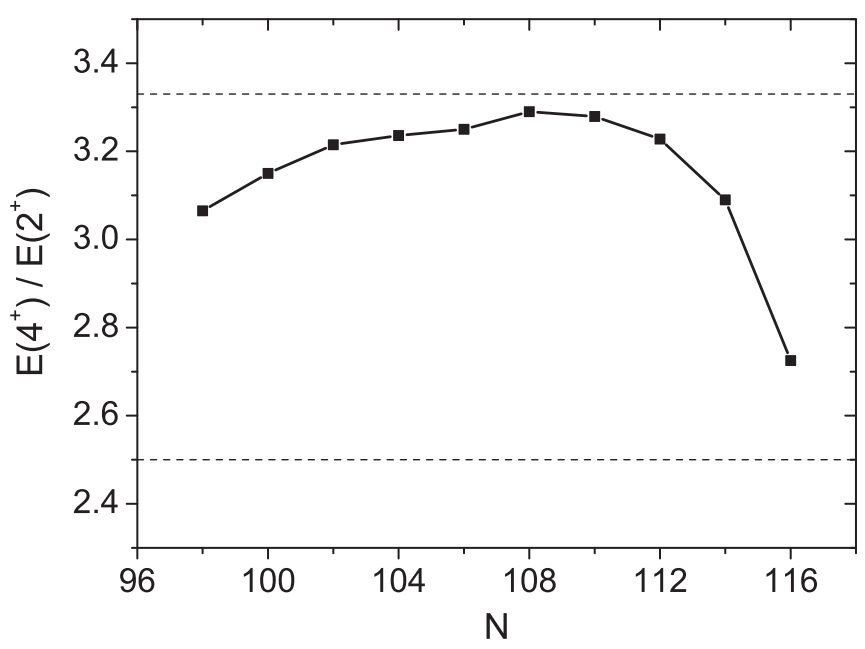

FIG. 1. Experimental ratio of the first $4^{+}$and $2^{+}$energies for $\mathrm{W}$ isotopic chain. Dashed horizontal lines are plotted at 3.33 and 2.5 for guidance, representing an axially deformed rotor and triaxial rotor, respectively .

\section{FORMALISM}

Most of the nuclei in the nuclear chart are deformed in their ground state. Therefore, it is more desirable to use the deformed mean-field solution as the starting basis, as the main parts of the correlations are taken into account in the deformed basis. Similarly to the conventional shell model, the PSM also works on the philosophy of the exact diagonalization of the effective many-body Hamiltonian. But unlike the shell model, PSM truncates the configuration space by selecting quasiparticle excitations efficiently [21]. To outline the PSM formalism briefly, at first the deformed quasiparticle basis is constructed with fixed deformation and axial symmetry through Nilsson-plus-BCS calculations. The basis truncation is done by taking multi-qp states near to the Fermi surfaces (both protons and neutrons). The choice of deformed mean-field basis essentially breaks the rotational symmetry in the many-body wave function. The broken symmetry is restored by performing angular-momentum projection (AMP). By means of AMP, one can obtain the sequence of good angular-momentum states which constitute the projected basis. Finally, the two-body Hamiltonian is exactly diagonalized in this projected basis.

The PSM wave function is a superposition of (angularmomentum) projected multi-qp states

$$
\left|\Psi_{I M}^{\sigma}\right\rangle=\sum_{K \kappa} f_{I K_{\kappa}}^{\sigma} \hat{P}_{M K}^{I}\left|\Phi_{\kappa}\right\rangle,
$$

where $\hat{P}_{M K}^{I}$ is the angular-momentum projection operator [27] and $\left|\Phi_{\kappa}\right\rangle$ denotes the qp basis. The deformed qp basis is constructed through Nilsson-BCS calculations by taking into account three major harmonic-oscillator shells, $N=4,5,6$ for neutrons and $N=3,4,5$ for protons. The multi-qp configurations up to 10-qp states for even-even nuclei are given by:

$$
\begin{gathered}
\left\{|\Phi\rangle, a_{v_{i}}^{\dagger} a_{v_{j}}^{\dagger}|\Phi\rangle, a_{\pi_{i}}^{\dagger} a_{\pi_{j}}^{\dagger}|\Phi\rangle, a_{v_{i}}^{\dagger} a_{v_{j}}^{\dagger} a_{\pi_{k}}^{\dagger} a_{\pi_{l}}^{\dagger}|\Phi\rangle,\right. \\
a_{v_{i}}^{\dagger} a_{v_{j}}^{\dagger} a_{v_{k}}^{\dagger} a_{v_{l}}^{\dagger}|\Phi\rangle, a_{\pi_{i}}^{\dagger} a_{\pi_{j}}^{\dagger} a_{\pi_{k}}^{\dagger} a_{\pi_{l}}^{\dagger}|\Phi\rangle,
\end{gathered}
$$




$$
\begin{aligned}
& a_{v_{i}}^{\dagger} a_{v_{j}}^{\dagger} a_{v_{k}}^{\dagger} a_{v_{l}}^{\dagger} a_{v_{m}}^{\dagger} a_{v_{n}}^{\dagger}|\Phi\rangle, a_{\pi_{i}}^{\dagger} a_{\pi_{j}}^{\dagger} a_{\pi_{k}}^{\dagger} a_{\pi_{l}}^{\dagger} a_{\pi_{m}}^{\dagger} a_{\pi_{n}}^{\dagger}|\Phi\rangle, \\
& a_{\pi_{i}}^{\dagger} a_{\pi_{j}}^{\dagger} a_{v_{k}}^{\dagger} a_{v_{l}}^{\dagger} a_{v_{m}}^{\dagger} a_{v_{n}}^{\dagger}|\Phi\rangle, a_{v_{i}}^{\dagger} a_{v_{j}}^{\dagger} a_{\pi_{k}}^{\dagger} a_{\pi_{l}}^{\dagger} a_{\pi_{m}}^{\dagger} a_{\pi_{n}}^{\dagger}|\Phi\rangle, \\
& a_{v_{i}}^{\dagger} a_{v_{j}}^{\dagger} a_{\pi_{k}}^{\dagger} a_{\pi_{l}}^{\dagger} a_{\pi_{m}}^{\dagger} a_{\pi_{n}}^{\dagger} a_{\pi_{o}}^{\dagger} a_{\pi_{p}}^{\dagger}|\Phi\rangle, a_{\pi_{i}}^{\dagger} a_{\pi_{j}}^{\dagger} a_{v_{k}}^{\dagger} a_{v_{l}}^{\dagger} a_{v_{m}}^{\dagger} a_{v_{n}}^{\dagger} a_{v_{o}}^{\dagger} a_{v_{p}}^{\dagger}|\Phi\rangle, \\
& a_{v_{i}}^{\dagger} a_{v_{j}}^{\dagger} a_{v_{k}}^{\dagger} a_{v_{l}}^{\dagger} a_{\pi_{m}}^{\dagger} a_{\pi_{n}}^{\dagger} a_{\pi_{o}}^{\dagger} a_{\pi_{p}}^{\dagger}|\Phi\rangle, \\
& a_{\pi_{i}}^{\dagger} a_{\pi_{j}}^{\dagger} a_{\pi_{k}}^{\dagger} a_{\pi_{l}}^{\dagger} a_{v_{m}}^{\dagger} a_{v_{n}}^{\dagger} a_{v_{o}}^{\dagger} a_{v_{p}}^{\dagger} a_{v_{q}}^{\dagger} a_{v_{r}}^{\dagger}|\Phi\rangle, \\
& \left.a_{v_{i}}^{\dagger} a_{v_{j}}^{\dagger} a_{v_{k}}^{\dagger} a_{v_{l}}^{\dagger} a_{\pi_{m}}^{\dagger} a_{\pi_{n}}^{\dagger} a_{\pi_{o}}^{\dagger} a_{\pi_{p}}^{\dagger} a_{\pi_{q}}^{\dagger} a_{\pi_{r}}^{\dagger}|\Phi\rangle\right\} .
\end{aligned}
$$

where $a_{\nu}^{\dagger}, a_{\pi}^{\dagger}\left(a_{v}, a_{\pi}\right)$ denote neutron and proton qp creation (annihilation) operators, respectively. $|\Phi\rangle$ in Eq. (2) represents the qp vacuum. It can be mentioned that the PSM works with multiple harmonic-oscillator shells for both neutrons and protons. The indices $v$ (for neutrons) and $\pi$ (for protons) in Eq. (2) are general; for example, a two-qp state can be of positive parity if both quasiparticles $i$ and $j$ are from the major $N$-shells that differ in $N$ by $\Delta N=0,2, \ldots$, or of negative parity if $i$ and $j$ are from those $N$-shells that differ by $\Delta N=1,3, \ldots$.

The energies and wave functions are obtained by solving the eigenvalue equation:

$$
\sum_{K^{\prime} \kappa^{\prime}}\left(H_{K \kappa, K^{\prime} \kappa^{\prime}}^{I}-E_{I}^{\sigma} N_{K \kappa, K^{\prime} \kappa^{\prime}}^{I}\right) f_{I K_{\kappa^{\prime}}^{\prime}}^{\sigma}=0,
$$

where $H_{K \kappa, K^{\prime} \kappa^{\prime}}^{I}$ and $N_{K \kappa, K^{\prime} \kappa^{\prime}}^{I}$ are, respectively, the projected matrix elements of the Hamiltonian and the norm

$$
H_{K \kappa, K^{\prime} \kappa^{\prime}}^{I}=\left\langle\Phi_{\kappa}\left|\hat{H} \hat{P}_{K K^{\prime}}^{I}\right| \Phi_{\kappa^{\prime}}\right\rangle, \quad N_{K \kappa, K^{\prime} \kappa^{\prime}}^{I}=\left\langle\Phi_{\kappa}\left|\hat{P}_{K K^{\prime}}^{I}\right| \Phi_{\kappa^{\prime}}\right\rangle .
$$

The main complexity in numerical calculations is to evaluate rotated matrix elements in the Hamiltonian and the norm

$$
\mathcal{H}_{\kappa \kappa^{\prime}}=\left\langle\Phi_{\kappa}|\hat{H}[\Omega]| \Phi_{\kappa^{\prime}}\right\rangle, \quad \mathcal{N}_{\kappa \kappa^{\prime}}=\left\langle\Phi_{\kappa}|[\Omega]| \Phi_{\kappa^{\prime}}\right\rangle,
$$

with the operator $[\Omega]$ defined as [21]

$$
[\Omega]=\frac{\hat{R}(\Omega)}{\langle\Phi|\hat{R}(\Omega)| \Phi\rangle} .
$$

$\hat{R}$ in the above equation is the rotation operator $[R(\Omega)=$ $\left.e^{-i \alpha J_{z}} e^{-i \beta J_{y}} e^{-i \gamma J_{z}}\right]$ and $\Omega$ the Euler angle. Since $\mathcal{H}_{\kappa \kappa^{\prime}}$ can be decomposed into terms expressed by the "linked" contraction and $\mathcal{N}_{\kappa \kappa^{\prime}}$ [21], the main task then concentrates on treating efficiently the rotated matrix element of the norm. For the sake of convenience, we rewrite $\mathcal{N}_{\kappa \kappa^{\prime}}$ as the following explicit form:

$$
\mathcal{N}_{\kappa \kappa^{\prime}}=\left\langle\Phi\left|a_{1} \cdots a_{n}[\Omega] a_{1^{\prime}}^{\dagger} \cdots a_{n^{\prime}}^{\dagger}\right| \Phi\right\rangle,
$$

which is usually evaluated $[21,28]$ by means of the generalized Wick's theorem. It was pointed out [29] that in applying the generalized Wick's theorem, a matrix element of Eq. (7) involving $n$ and $n^{\prime}$ qps, respectively on the left and right sides of $[\Omega]$, contains $\left(n+n^{\prime}-1\right)$ !! terms. In practice, the number of terms becomes so large that it is very difficult to write down expressions explicitly for more than four-qp states. Recently, by means of fermion coherent states and the Grassmann integral, the Pfaffian formulas have been proposed [29] to calculate the rotated matrix element. The proposal was largely inspired by the initial introduction of the Pfaffian idea by Robledo [30] to treat the sign problem in calculations of the overlap of Hartree-Fock-Bogoliubov (HFB) wave-functions. It was realized later [31-35] that the method can be applied to evaluation of general HFB matrix elements. The new Pfaffian method has turned out to be a feasible and very efficient algorithm for calculations with projected multi-qp states. The first PSM calculations using the Pfaffian formulas have been published with examples of studying high-spin states [23,24].

The PSM employs the Hamiltonian with separable forces:

$$
\hat{H}=\hat{H}_{0}-\frac{1}{2} \chi_{Q Q} \sum_{\mu} \hat{Q}_{2 \mu}^{\dagger} \hat{Q}_{2 \mu}-G_{M} \hat{P}^{\dagger} \hat{P}-G_{Q} \sum_{\mu} \hat{P}_{2 \mu}^{\dagger} \hat{P}_{2 \mu}
$$

where $\hat{H}_{0}$ is the spherical single-particle term including the spin-orbit force [36] and the rest is the quadrupole+pairing type of interactions, which contains three parts. The strength of the quadrupole-quadrupole term $\chi_{Q Q}$ is determined in a selfconsistent manner so that it is related to the deformation of the basis [21]. In the present work, the monopole-pairing strength is taken to be of the form $G_{M}=\left[G_{1} \mp G_{2}(N-Z) / A\right] / A$, where "+" ("-") is for protons (neutrons), with $G_{1}=21.24$ and $G_{2}=13.86$ (both in $\mathrm{MeV}$ ) being the coupling constants. The quadrupole-pairing strength $G_{Q}$ is taken, as usual [21], to be $16 \%$ of $G_{M}$ for all the nuclei considered in this study.

\section{RESULT AND DISCUSSION}

\section{A. Deformed basis, quasiparticle configurations, and band diagram}

Microscopic descriptions for the rotational motion of a stably deformed nucleus involve coherent contributions from many nucleons. The yrast state is of particular interest because it carries valuable information of how the nucleons are organized in the lowest energy state for a given angular momentum and how the organization responds to the rotation. In the ground state of even-even nuclei, nucleons are coupled pairwise. The (angular-momentum) projected qp vacuum state, the first term in (2), describes the rotational behavior of the ground band in a deformed nucleus. As the nucleus rotates, the Coriolis force, acting on the nucleon pairs in the intrinsic rotating frame, can break the pairs and thus destroy the nuclear superfluidity. A sudden increase in moment of inertia (MoI) at a given angular momentum is usually an indication of the pair breaking with the spins of the nucleons aligned along the axis of rotation. The projected two-qp configurations in (2) describe such spin-aligned states [37]. Mixing of the projected qp vacuum and projected two-qp states through diagonalization describes the interplay between the collective ground state and the spin-aligned ones. In this way, the experimentally observed changes in MoI in many well-deformed nuclei can be described microscopically.

For each $\mathrm{W}$ isotope, we assume an axially symmetric basis with prolate deformation and construct the deformed basis with quadrupole $\varepsilon_{2}$ and hexadecapole $\varepsilon_{4}$ deformation parameters, which are taken initially from the tables [38] as guidance and adjusted slightly, to generate a suitable basis. The parameters are listed in Table I, where the decreasing trend in $\varepsilon_{2}$ and increasing trend in $\varepsilon_{4}$ as the neutron number increases (see Ref. [38]) are reflected. As we shall demonstrate, they are 
TABLE I. Quadrupole and hexadecapole deformation parameters for the deformed bases employed in the calculation.

\begin{tabular}{lccccccc}
\hline \hline & ${ }^{174} \mathrm{~W}$ & ${ }^{176} \mathrm{~W}$ & ${ }^{178} \mathrm{~W}$ & ${ }^{180} \mathrm{~W}$ & ${ }^{182} \mathrm{~W}$ & ${ }^{184} \mathrm{~W}$ & ${ }^{186} \mathrm{~W}$ \\
\hline$\varepsilon_{2}$ & 0.262 & 0.220 & 0.220 & 0.210 & 0.230 & 0.210 & 0.200 \\
$\varepsilon_{4}$ & 0.033 & 0.047 & 0.025 & 0.020 & 0.020 & 0.040 & 0.060 \\
\hline \hline
\end{tabular}

good starting bases for both the low-lying and high-spin states in which the rotational motion and qp excitations compete near the yrast line. Here a slight adjustment from the deformation parameters given in Ref. [38] is introduced because these deformation parameters are optimal for the ground state but may not be the best when many multi-qp bands are studied. We note that a conceptually advanced, but technically complicated, treatment is to include in the basis qp-configurations belonging to different deformations, as in the examples recently shown by Chen and Egido [39].

Pairing correlations are treated by BCS calculations. The pairing energy gaps are determined by the pairing force strengths (given at the end of Sec. II) and by the actual level distribution in the standard BCS calculation by solving the gap equation. In Table II, we show the resulting pairing-gap parameters for each isotope. It should be noted that the unperturbed bandhead energies of high- $K$ isomeric states depend on the size of pairing gaps in the single-particle spectrum. As one can see from Table II, the calculated neutron pairing gaps become smaller as the neutron number goes up, so that one expects the occurrence of low-energy qp isomers in heavier isotopes ( $\mathrm{such}$ as ${ }^{184,186} \mathrm{~W}$ ). We point out that the values in Table II are by $20-30 \%$ smaller than the even-odd mass differences calculated from the neutron and proton separation energies [40], which is nevertheless necessary to reproduce those rather low excitation-energies of the multi-qp $K$-isomeric bands.

The calculation of multi-qp energies for the configurations in (2) gives us the information where these states lie relative to the ground state, so that one can identify those low-lying states in each configuration for the diagonalization procedure. Equivalently, this enables us to truncate the shell-model space under the physical guidance explained in Ref. [22]. Thus shell-model truncation is implemented simply by excluding the states with higher energies in each configuration. For the present calculation, the number of projected qp-states, i.e., the dimension of the summation in Eq. (1), is usually a few hundred. To give an example for the highest dimension involved in the present paper, the ${ }^{174} \mathrm{~W}$ calculation uses energy cutoffs of $3.5 \mathrm{MeV}$ for 2-qp, 4.5 MeV for 4-qp, 5.2 MeV for 6-qp, 8.6 MeV for 8-qp, and 10.0 MeV for 10-qp states. The resulting numbers of multi-qp configurations are 77 for

TABLE II. Calculated neutron and proton pairing-gap parameters (in $\mathrm{MeV}$ ) for the qp-vacuum states.

\begin{tabular}{lccccccc}
\hline \hline & ${ }^{174} \mathrm{~W}$ & ${ }^{176} \mathrm{~W}$ & ${ }^{178} \mathrm{~W}$ & ${ }^{180} \mathrm{~W}$ & ${ }^{182} \mathrm{~W}$ & ${ }^{184} \mathrm{~W}$ & ${ }^{186} \mathrm{~W}$ \\
\hline Neutron & 0.760 & 0.840 & 0.684 & 0.551 & 0.575 & 0.617 & 0.584 \\
Proton & 0.740 & 0.663 & 0.699 & 0.706 & 0.710 & 0.672 & 0.647 \\
\hline \hline
\end{tabular}

2-qp, 210 for 4-qp, 260 for 6-qp, 109 for 8-qp, and 23 for 10 -qp states. Together with the 0-qp state, the total number of included qp states in this example is 680 .

The (angular-momentum-projected) multi-qp configurations in PSM form a shell-model basis in which a twobody Hamiltonian is diagonalized. Each of these multi-qp configurations carries a total $K$ quantum number as a sum of individual $K$ from each qp state. Therefore, one can classify the observed states using the quantum number $K$, although the diagonalization would mix the multi-qp $K$ configurations in a process known as $K$ mixing.

The energy of a $K$ band as a function of spin $I$ is expressed as

$$
E_{\kappa}(I)=\frac{\left\langle\Phi_{\kappa}\left|\hat{H} \hat{P}_{K K}^{I}\right| \Phi_{\kappa}\right\rangle}{\left\langle\Phi_{\kappa}\left|\hat{P}_{K K}^{I}\right| \Phi_{\kappa}\right\rangle}
$$

The diagram representing variation of rotational energies of various bands with spin $I$ is referred to as a band diagram [28]. As each lowest-lying band corresponds roughly to a set of observable energy levels of the same structure, one can discuss a band diagram and learn the main band structures even before diagonalization. The angular-momentum-projected energies for various qp configurations are shown in Fig. 2 for the W isotopic chain investigated in the present study.

It is seen in Fig. 2 that for all the nuclei shown here, bands from different configurations cross with each other at different angular momenta. The physics behind these crossings is that some multi-qp bands that lie higher in energy when they begin to rotate can lower their energy and dive into the yrast region at higher angular momenta. In this way, they may cross several lower-order of multi-qp bands and eventually become the lowest at certain angular momentum and, therefore, are the most important components for the yrast band. As discussed in Ref. [37], the crossing angle of the two crossing bands can have a consequence in the change of MoI. A large crossing angle corresponding to a sharp band crossing results in a large disturbance in MoI, while a small crossing angle corresponding to a gentle band crossing leads to a small or nondisturbance in MoI. In Fig. 2, the general picture is that sharper crossings occur mainly at lower spins up to $\sim 20 \hbar$. We thus expect significant changes in MoI will be seen before $\sim 20 \hbar$. After that spin bands still cross with each other but with smaller crossing angles. Much denser band distributions are seen in the high-spin region in Fig. 2, indicating complex mixings of different configurations. As a representative example, the dominant configurations that contribute to the yrast band at different spin intervals of ${ }^{174} \mathrm{~W}$ are given in Table III.

\section{B. Rotational behavior: Moments of inertia of the yrast bands}

For a nucleus in its ground state, the paring force favors the like nucleons to form pairs and the system behaves like a superfluid. In rotating nuclei, the Coriolis force becomes stronger and tends to break the pairs and align the single particle angular momentum along the axis of rotation. The Coriolis force acts differently on different orbitals and depends on the the size of the single-particle angular momentum $j$ 

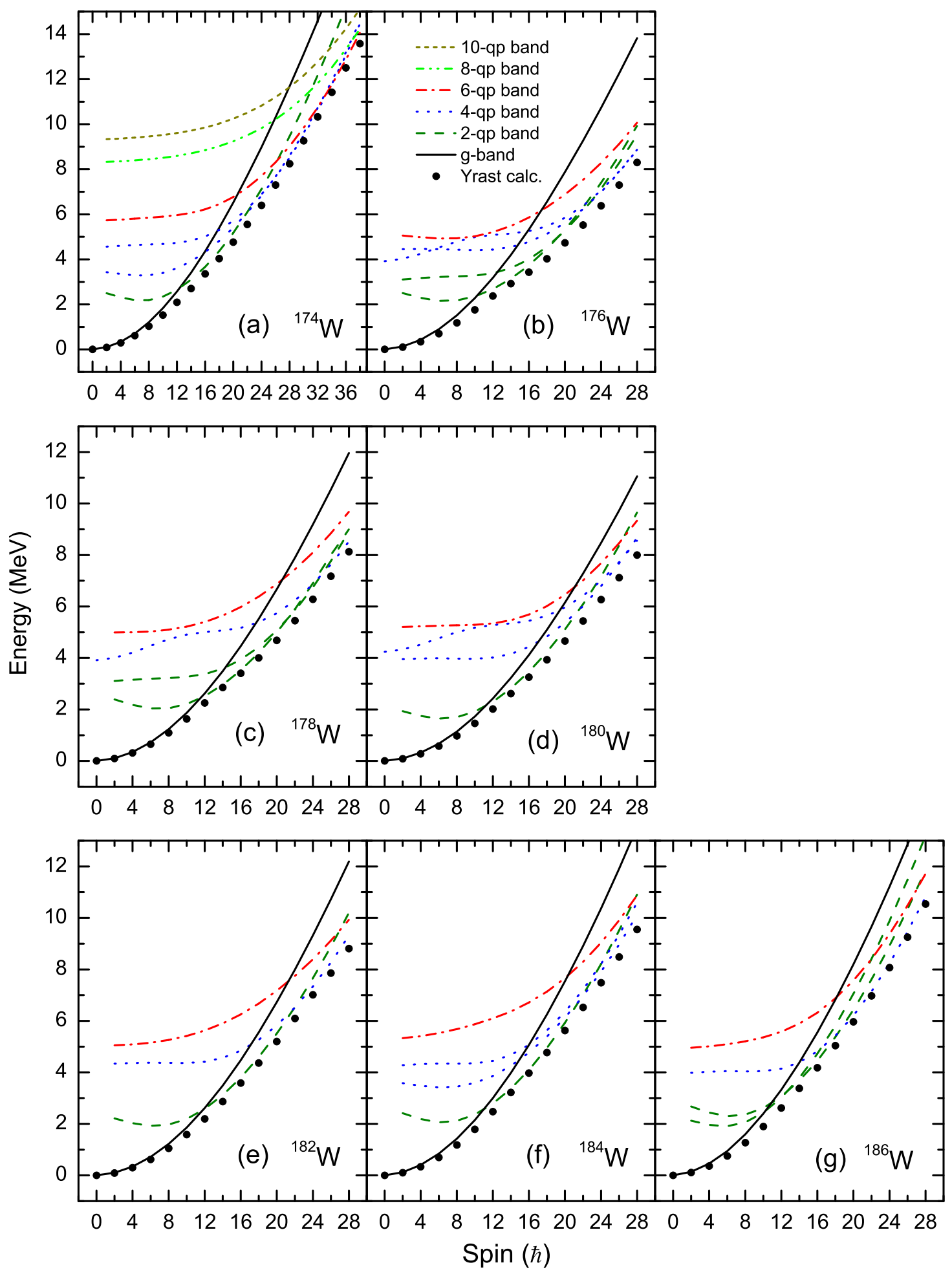

FIG. 2. Band diagrams for (a) ${ }^{174} \mathrm{~W}$, (b) ${ }^{176} \mathrm{~W}$, (c) ${ }^{178} \mathrm{~W}$, (d) ${ }^{180} \mathrm{~W}$, (e) ${ }^{182} \mathrm{~W}$, (f) ${ }^{184} \mathrm{~W}$, and (g) ${ }^{186} \mathrm{~W}$. Note that only even-spin states are plotted for clarity in these curves. See the text for details.

of a nucleon under consideration, which is known as the Stephens-Simon effect [41].

Structural changes along a band can be better understood with more sensitive plots of kinematical MoI, defined as $\mathcal{J}(I)=(2 I-1) / E_{\gamma}(I)$, where $E_{\gamma}(I)=E(I)-E(I-2)$ is the transition energy. It is instructive to analyze the MoI variations in the yrast bands to learn about the rotational response of the systems. The calculated MoI for the yrast bands are shown in Fig. 3 for ${ }^{174-186} \mathrm{~W}$ and compared with available experimental data. An overall good reproduction of 
TABLE III. Main configurations that contribute to the yrast band at different spin intervals of ${ }^{174} \mathrm{~W}$. In the first column, the spin indicates the approximate spin value from which the corresponding configuration begins to play a role.

\begin{tabular}{cccc}
\hline \hline Spin & Type & $K^{\pi}$ & Configuration \\
\hline 10 & $2-q p$ & $1^{+}$ & $\nu 5 / 2^{+}[642] \otimes 7 / 2^{+}[633]$ \\
18 & $4-q p$ & $2^{+}$ & $\nu 5 / 2^{+}[642] 7 / 2^{+}[633] \otimes \pi 1 / 2^{-}[541] 3 / 2^{-}[532]$ \\
20 & $4-q p$ & $1^{+}$ & $\nu 5 / 2^{+}[642] 7 / 2^{+}[633] \otimes \pi 1 / 2^{-}[541] 1 / 2^{-}[541]$ \\
28 & $6-q p$ & $2^{+}$ & $\nu 5 / 2^{+}[642] 7 / 2^{+}[633] \otimes \pi 1 / 2^{-}[541] 3 / 2^{-}[532] 9 / 2^{-}[514] 9 / 2^{-}[514]$ \\
34 & $8-q p$ & $2^{+}$ & $\nu 3 / 2^{+}[651] 5 / 2^{+}[642] 7 / 2^{+}[633] 7 / 2^{+}[633] \otimes \pi 1 / 2^{-}[541] 1 / 2^{-}[541] 7 / 2^{-}[523] 9 / 2^{-}[514]$ \\
38 & $10-q p$ & $2^{+}$ & $v 3 / 2^{+}[651] 5 / 2^{+}[642] 7 / 2^{+}[633] 7 / 2^{+}[633] \otimes \pi 1 / 2^{-}[541] 1 / 2^{-}[541] 7 / 2^{-}[523] 9 / 2^{-}[514] \otimes \pi 5 / 2^{+}[402] 5 / 2^{+}[402]$ \\
\hline \hline
\end{tabular}

the experimental MoI is obtained by the PSM calculations. The curves show a general trend of the increase in MoI in the low-spin region but the MoI remains stable (in some cases nearly constant) in the high-spin region.
The behavior of MoI in the $\mathrm{W}$ isotopes can be understood from the discussion about band crossings in the last section. For the even-even rare-earth nuclei studied here, the neutron Fermi surface is mainly built on the $i_{13 / 2}$ orbital. The $i_{13 / 2}$ orbital is
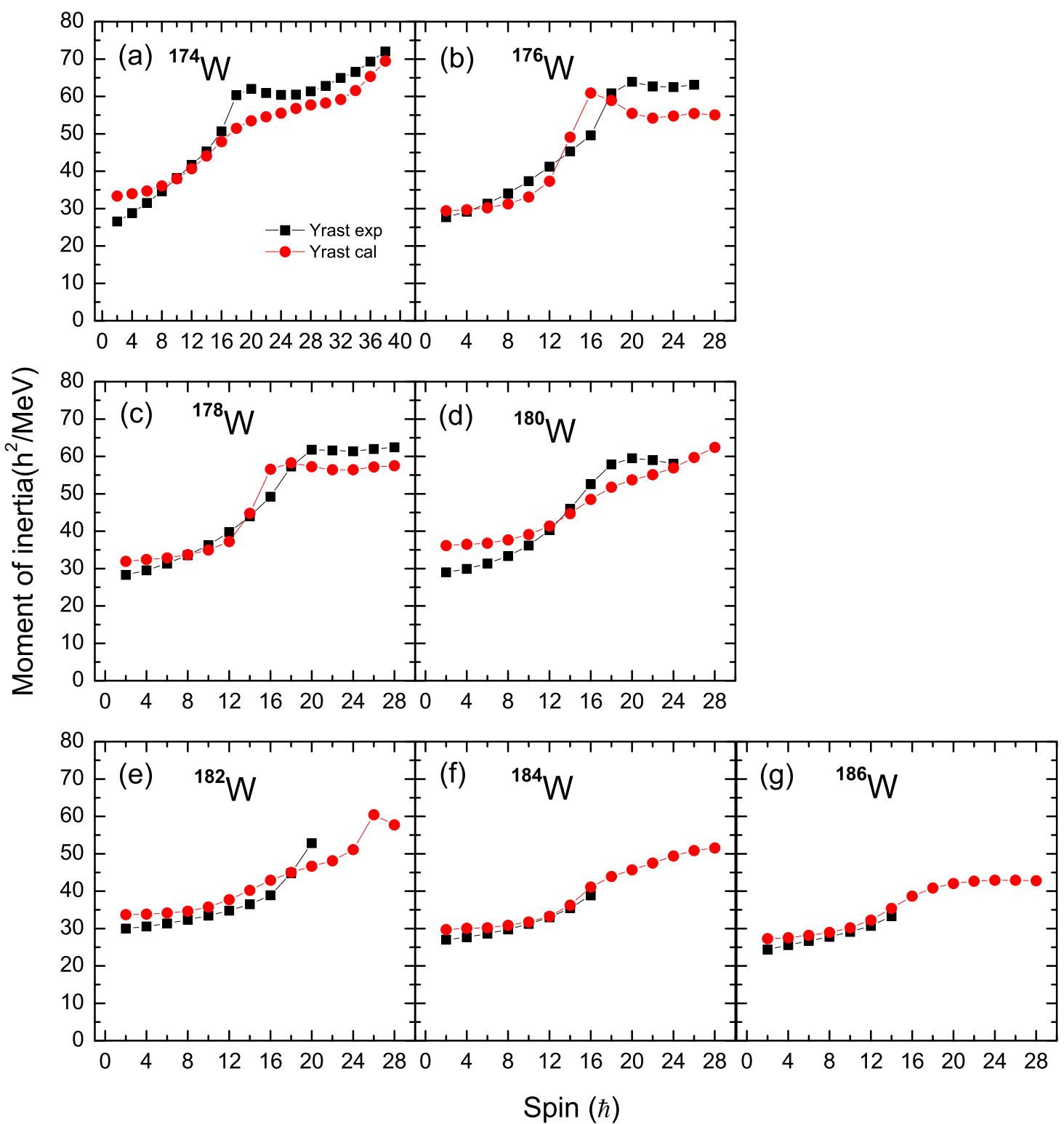

FIG. 3. Calculated moments of inertia for the yrast band of (a) ${ }^{174} \mathrm{~W}$, (b) ${ }^{176} \mathrm{~W}$, (c) ${ }^{178} \mathrm{~W}$, (d) ${ }^{180} \mathrm{~W}$, (e) ${ }^{182} \mathrm{~W}$, (f) ${ }^{184} \mathrm{~W}$, and (g) ${ }^{186} \mathrm{~W}$. 


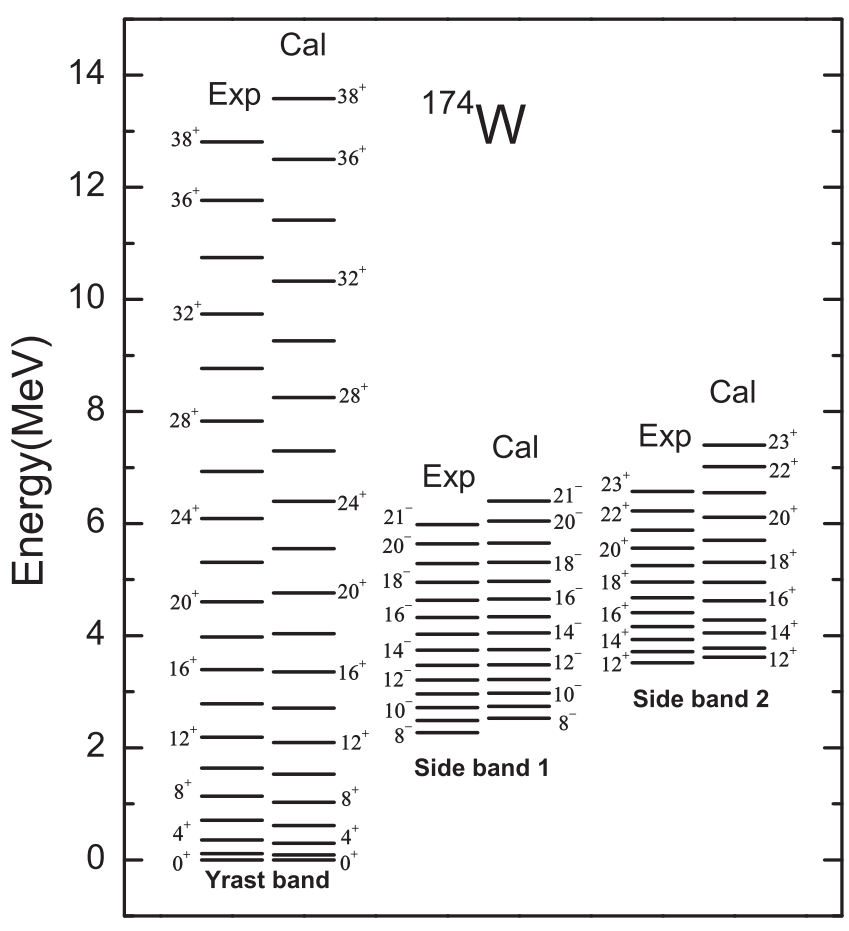

FIG. 4. Comparison of calculated energies with available data for ${ }^{174}$ W. Experimental values are taken from Refs. [42,44,45].

well separated from the same parity orbitals and often denoted as pure- $j$ states. Also the component states from higher- $j$ orbitals do not Coriolis mix much with other- $j$ states. Due to the rotational motion, the first pair breaking gives rise to a 2-quasineutron (based on $i_{13 / 2}$ ) band. The low and medium spin structures of these nuclei are mainly governed by the interplay of the ground band and the 2-quasineutron band. As the nucleus rotates faster, more higher-qp configurations come into play. In lighter-mass $\mathrm{W}$ isotopes, rapid increases in the MoI are observed around the spin range 10-16 $\hbar$. As shown in Fig. 2, in the lighter mass nuclei, the crossing angle between the zero-qp configuration (i.e., ground band) and the two-qp configuration is larger. In other words, we can say that the interaction between the ground band and the $s$ band is small and hence gives rise to a sharp increase in the MoI at the crossing.

\section{Energy levels}

Both positive- and negative-parity energy levels are obtained by diagonalizing the Hamiltonian (8) in the projected basis (2). The calculated energy levels of even-even ${ }^{174-186} \mathrm{~W}$ along with available experimental data are plotted in Figs. 4-10.

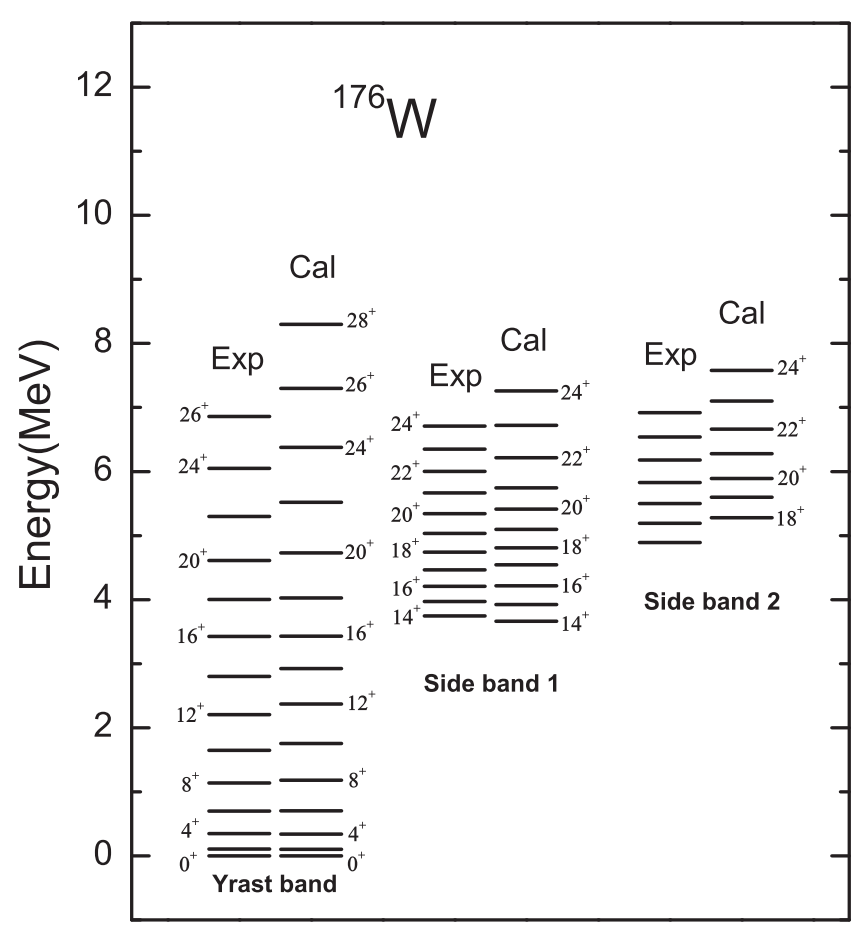

FIG. 5. Comparison of calculated energies with available data for ${ }^{176} \mathrm{~W}$. Experimental values are taken from Refs. [46,47].

$$
\text { 1. }{ }^{174} \mathrm{~W}
$$

The rotating nucleus ${ }^{174} \mathrm{~W}$ exhibits axially prolate shape for a wide range of rotational frequencies $[42,43]$. The yrast band has been established to $38 \hbar$ [44]. From Fig. 4, we can see that the experimental yrast band is quite well reproduced with PSM calculations. This nucleus is also characterized by low- $K$ and high- $K$ bands up to highly excited states, $39 \hbar$ [44]. In particular, two high- $K$ bands based on $K=8$ and $K=12$ isomeric states with half-lives of $158 \mathrm{~ns}$ and $128 \mathrm{~ns}$ have been observed [42]. From PSM analysis, we see that the $K=8$ isomeric state has a two-qp structure with a dominant configuration of $\pi 7 / 2^{+}[404] \otimes \pi 9 / 2^{-}$[514]. The isomeric band based on $K=8^{-}$is experimentally known up to $21 \hbar$ and well accounted in our PSM calculation ("sideband 1" in Fig. 4) except the bandhead energy is over estimated by about $200 \mathrm{keV}$. The $K=12$ isomeric state has four-qp structure with the dominant configuration listed in Table IV, which is reasonably described by the calculation except for the highest spin states.

\section{2. ${ }^{176} \mathrm{~W}$}

The calculated energy levels along with experimental data are shown in Fig. 5 for ${ }^{176} \mathrm{~W}$. The experimental yrast band

TABLE IV. Main configurations of the side bandheads of ${ }^{174} \mathrm{~W}$.

\begin{tabular}{lccc}
\hline \hline Band & Type & $K^{\pi}$ & Configuration \\
\hline Side 1 & Two-qp & $8^{-}$ & $\pi 7 / 2^{+}[404] \otimes 9 / 2^{-}[514]$ \\
Side 2 & Four-qp & $12^{+}$ & $v 1 / 2^{-}[521] 7 / 2^{+}[633] \otimes \pi 7 / 2^{+}[404] 9 / 2^{-}[514]$ \\
\hline \hline
\end{tabular}


TABLE V. Main configurations of the side bandheads of ${ }^{176} \mathrm{~W}$.

\begin{tabular}{lllc}
\hline \hline Band & Type & $K^{\pi}$ & Configuration \\
\hline Side 1 & Four-qp & $14^{+}$ & $v 5 / 2^{-}[512] 7 / 2^{+}[633] \otimes \pi 7 / 2^{+}[404] 9 / 2^{-}[514]$ \\
Side 2 & Six-qp & $18^{+}$ & $v 5 / 2^{-}[512] 7 / 2^{-}[514] 1 / 2^{-}[521] 7 / 2^{+}[633] \otimes \pi 7 / 2^{+}[404] 9 / 2^{-}[514]$ \\
\hline \hline
\end{tabular}

is established up to $I=26 \hbar[46,47]$. Crowell et al. [46] observed a high- $K$ isomer with $I, K^{\pi}=14,14^{+}$and $t_{1 / 2}=$ $35 \pm 10 \mathrm{~ns}$. They predicted the isomeric state to be four$\mathrm{qp}$ in nature with configuration $v 5 / 2^{-}[512] \otimes v 7 / 2^{+}[633] \otimes$ $\pi 7 / 2^{+}[404] \otimes \pi 9 / 2^{-}[514]$. A similar isomer is also known to exist in the neighboring even-even isotone ${ }^{174} \mathrm{Hf}$ [48]. In both cases severe breakdown of the $K$-selection rule has been observed. Our PSM calculations describe this isomeric state and the band based on it reasonably well. Additionally, Crowell et al. [46] observed a band based on a 10-ns isomer with a bandhead energy of $4.893 \mathrm{MeV}$. The spin and parity of the 10-ns isomer are not known experimentally. Our calculations indicate that this band may be built on a six-qp isomer with $K^{\pi}=18^{+}$. Despite the small discrepancy between the calculated and experimental bandhead energies, the moment of inertia is reproduced quite well by PSM calculations. We suggest the dominant configuration of the band as $v 5 / 2^{-}[512] \otimes v 7 / 2^{-}[514] \otimes v 1 / 2^{-}[521] \otimes$ $v 7 / 2^{+}[633] \otimes \pi 7 / 2^{+}[404] \otimes \pi 9 / 2^{-}[514]$. The suggested configurations for these two isomeric bands are listed in Table V.

\section{3. ${ }^{178} \mathrm{~W}$}

The structure of $N=104$ nuclei plays an important role as these nuclei are marked as the boundary between some vibrational modes and rotation-aligned excitations [51]. The macroscopic-microscopic calculations [38] show that in this region the maximum deformation occurs at $N=104$ and $Z=66$. In fact, ${ }^{170}$ Dy represents the valence maximum (i.e., proton-neutron valence product $N_{p} N_{n}$ ) in this region. However, the experimental results [52] do not support this idea. Recent theoretical calculation predicts a deformed shell closure at $N=100$ [53]. This has also been experimentally verified recently [54] for Sm and Gd nuclei. This scenario may get changed if one moves towards the higher side of the rare-earth region. The quasiparticle alignment hypothesis is necessarily valid for the ${ }^{178} \mathrm{~W}(N=104)$ isotope. The PSM calculations assuming axial symmetry in the basis remain successful in describing the yrast and $K$-isomeric bands, as shown in Fig. 6.

Apart from the yrast band, several multi-quasiparticle high- $K$ bands are known in ${ }^{178} \mathrm{~W}$. Two 2-quasineutron bands ("sideband 1" and "sideband 2" in Fig. 6) with configurations $v 5 / 2^{-}[512] \otimes v 7 / 2^{-}[514]$ and $v 7 / 2^{-}[514] \otimes v 7 / 2^{+}[633]$, respectively, are obtained from PSM calculations. The configuration assignments are consistent with the experimental suggestions [49]. A negative-parity band based on a 3-ns, $K=14$ isomer is also known. However, the experimental band is established up to spin $15 \hbar$ only. From calculations, we confirmed that this band has the four-qp configuration of $v 5 / 2^{-}[512] \otimes v 7 / 2^{-}[514] \otimes \pi 7 / 2^{+}[404] \otimes \pi 9 / 2^{-}[514]$. The positive-parity four-qp particle band ("sideband 4" in Fig. 6) based on $K=15$ is known up to very high spin states of $39 \hbar$. Similarly to the experimental result [49], we also obtained the dominant configuration for the bandhead as $v 7 / 2^{-}[514] \otimes v 7 / 2^{+}[633] \otimes \pi 7 / 2^{+}[404] \otimes \pi 9 / 2^{-}[514]$. The main configurations for each band obtained from the calculations are listed in Table VI.

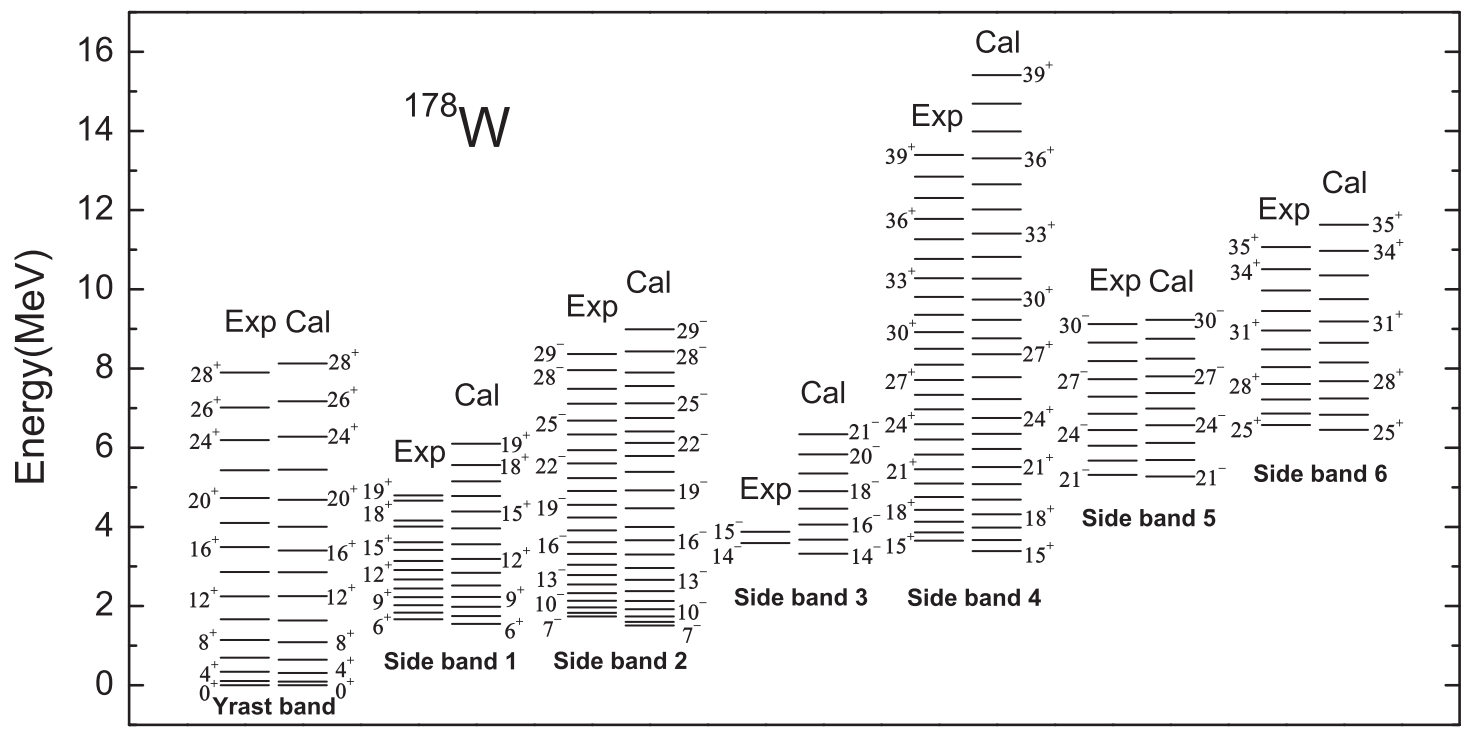

FIG. 6. Comparison of calculated energies with available data for ${ }^{178} \mathrm{~W}$. Experimental values are taken from Refs. [49,50]. 
TABLE VI. Main configurations of the side bandheads of ${ }^{178} \mathrm{~W}$.

\begin{tabular}{lccc}
\hline \hline Band & Type & $K^{\pi}$ & Configuration \\
\hline Side 1 & Two-qp & $6^{+}$ & $v 5 / 2^{-}[512] \otimes 7 / 2^{-}[514]$ \\
Side 2 & Two-qp & $7^{-}$ & $v 7 / 2^{-}[514] \otimes 7 / 2^{+}[633]$ \\
Side 3 & Four-qp & $14^{-}$ & $v 5 / 2^{-}[512] 7 / 2^{-}[514] \otimes \pi 7 / 2^{+}[404] 9 / 2^{-}[514]$ \\
Side 4 & Four-qp & $15^{+}$ & $v 7 / 2^{-}[514] 7 / 2^{+}[633] \otimes \pi 7 / 2^{+}[404] 9 / 2^{-}[514]$ \\
Side 5 & Six-qp & $21^{-}$ & $\nu 5 / 2^{-}[512] 7 / 2^{-}[514] 7 / 2^{+}[633] 9 / 2^{+}[624] \otimes \pi 5 / 2^{+}[402] 9 / 2^{-}[514]$ \\
Side 6 & Eight-qp & $25^{+}$ & $v 5 / 2^{-}[512] 7 / 2^{-}[514] 7 / 2^{+}[633] 9 / 2^{+}[624] \otimes \pi 1 / 2^{-}[541] 5 / 2^{+}[402] 7 / 2^{+}[404] 9 / 2^{-}[514]$ \\
\hline \hline
\end{tabular}

${ }^{178} \mathrm{~W}$ is one of the few examples where high- $K$ bands with more than six quasiparticles are observed. A six-qp $K^{\pi}=21^{-}$ band with configuration $v 5 / 2^{-}[512] \otimes v 7 / 2^{-}[514] \otimes$ $v 7 / 2^{+}[633] \otimes v 9 / 2^{+}[624] \otimes \pi 5 / 2^{+} \quad[402] \otimes \pi 9 / 2^{-}[514]$ and an eight-qp $K^{\pi}=25^{+}$band with configuration $v 5 / 2^{-}[512] \otimes v 7 / 2^{-}[514] \otimes v 7 / 2^{+}[633] \otimes v 9 / 2^{+} \quad[624] \otimes$ $\pi 1 / 2^{-}[541] \otimes \pi 5 / 2^{+}[402] \otimes \pi 7 / 2^{+}[404] \otimes \pi 9 / 2^{-}$ are reported in Ref. [49]. As one can see in Fig. 6, both the bands including bandhead energies are well accounted for in PSM calculations up to known spin levels.

$$
\text { 4. }{ }^{180} \mathrm{~W}
$$

A comparison of calculated results and experimental data for ${ }^{180} \mathrm{~W}$ is illustrated in Fig. 7 with the suggested main configuration for each band listed in Table VII. The yrast band is experimentally established up to $24 \hbar$. A $K=5^{-}$isomeric band is observed experimentally and known up to spin $I=9 \hbar$ [55]. From experiment, it was suggested that this band has a significant 2-qp component with the neutron configuration $v 1 / 2^{-}[521] \otimes v 9 / 2^{+}[624]$. By analyzing the quasiparicle configurations of PSM, we found that two possible 2qpconfigurations, $v 1 / 2^{-}[521] \otimes v 9 / 2^{+}[624]$ (bandhead energy of $2.022 \mathrm{MeV}$ ) and $\pi 1 / 2^{+}[411] \otimes \pi 9 / 2^{-}$[514] (bandhead energy of $1.495 \mathrm{MeV}$ ), can form the $5^{-}$configuration. Although both of them have quite similar MoI trends, the proton 2qpconfiguration is energetically more favorable. Therefore, we suggest that this 2-qp band has dominant proton 2-qp structure with configuration $\pi 1 / 2^{+}[411] \otimes \pi 9 / 2^{-}$[514]. To determine the configuration experimentally, $g$-factor measurements on this band are desired as states based on proton and neutron structures can have very different $g$-factors.

A two-qp band based on the $K=8^{-}, 5.47-\mathrm{ms}$ isomer is known from an early experiment [59]. It is suggested that this isomer involves the configuration of $v 7 / 2^{-}[514] \otimes$ $\nu 9 / 2^{+}[624]$, but has the possibility of mixing of two-neutron and the two-proton configurations. In the PSM calculation we found two two-qp configurations that may contribute to form this state. One has dominant two-quasineutron structure with configuration of $v 7 / 2^{-}[514] \otimes v 9 / 2^{+}[624]$, and the other has two-quasiproton structure with $\pi 7 / 2^{+}[404] \otimes \pi 9 / 2^{-}[514]$ as the dominant configuration. Both the theoretical bands based on $\mathrm{K}=8^{-}$states have very similar $\mathrm{MoI}$ and both are also energetically close to the experimental band. We note that in the isotone ${ }^{178} \mathrm{Hf}$, the picture of two-proton/two-neutron mixing has been well established [60]. In order to finally assign the configuration of the $K=8^{-}$isomer in ${ }^{180} \mathrm{~W}$, again $g$-factor measurements of this state are desired.

In Ref. [61], the $K=8^{+}$band that rotates with a titled axis (the so-called $t$ band) was observed. This $t$ band was

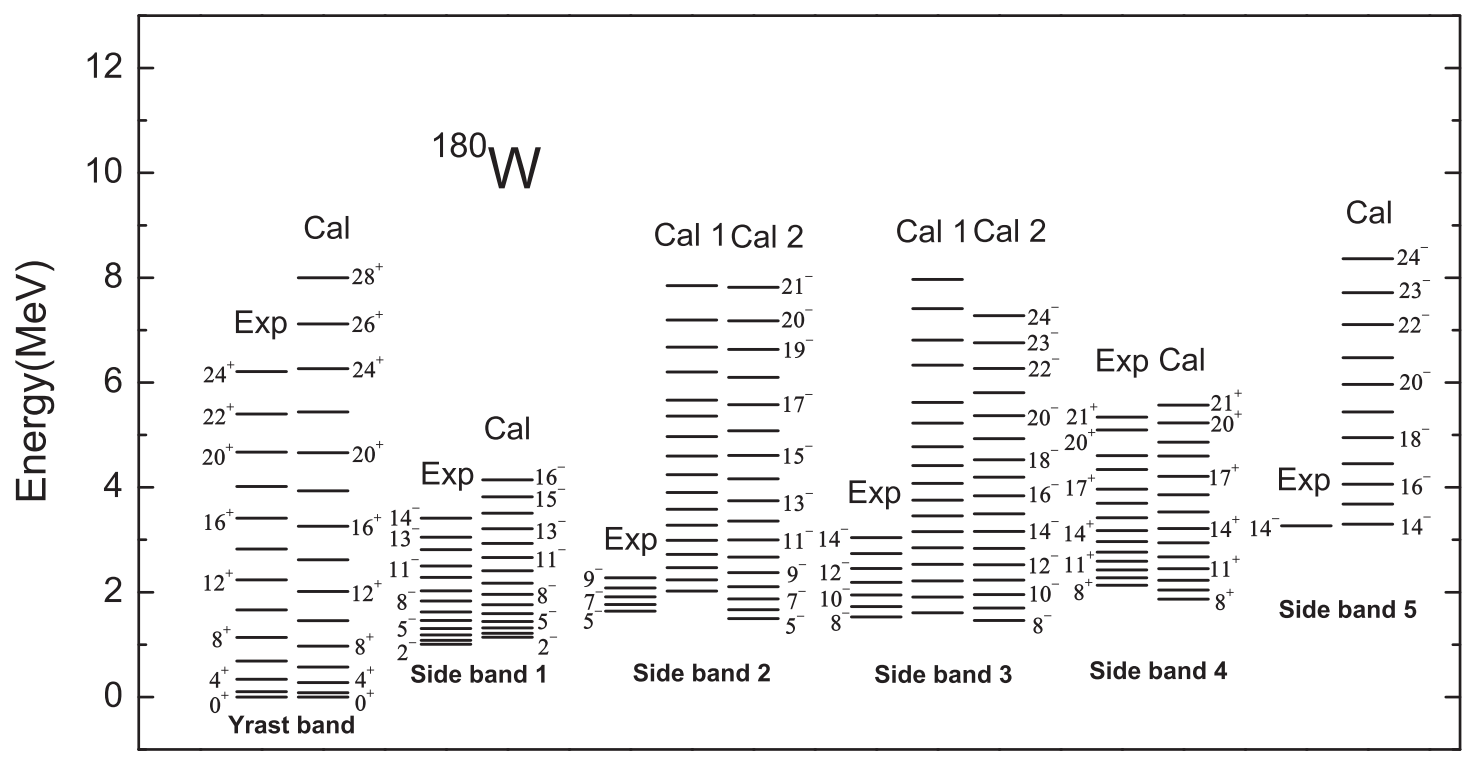

FIG. 7. Comparison of calculated energies with available data for ${ }^{180} \mathrm{~W}$. Experimental values are taken from Ref. [55]. 
TABLE VII. Main configurations of the side bandheads of ${ }^{180} \mathrm{~W}$.

\begin{tabular}{llll}
\hline \hline Band & Type & $K^{\pi}$ & Configuration \\
\hline Side 1 & Two-qp & $2^{-}$ & $\pi 5 / 2^{+}[402] \otimes 9 / 2^{-}[514]$ \\
Side 2 cal 1 & Two-qp & $5^{-}$ & $\nu 1 / 2^{-}[521] \otimes 9 / 2^{+}[624]$ \\
Side 2 cal 2 & Two-qp & $5^{-}$ & $\pi / 2^{+}[411] \otimes 9 / 2^{-}[514]$ \\
Side 3 cal 1 & Two-qp & $8^{-}$ & $\pi 7 / 2^{-}[514] \otimes 9 / 2^{+}[624]$ \\
Side 3 cal 2 & Two-qp & $8^{-}$ & $\pi 7 / 2^{+}[404] \otimes 9 / 2^{-}[514]$ \\
Side 4 & Two-qp & $8^{+}$ & $\nu 7 / 2^{+}[633] \otimes 9 / 2^{+}[624]$ \\
Side 5 & Four-qp & $14^{-}$ & $\nu 7 / 2^{-}[514] 9 / 2^{+}[624] \otimes \pi 5 / 2^{+}[402] 7 / 2^{+}[404]$ \\
\hline \hline
\end{tabular}

calculated [62] by PSM and discussed as an interplay with the low- $K$ bands that contribute to the yrast structure. In Fig. 7, we also include the results for this $K=8^{+}$band. As one can see, the position of the band is correctly reproduced, although the calculated level distribution is a bit more spread than the data.

For the four-qp $K=14^{-}$isomer in ${ }^{180} \mathrm{~W}$, from experiment it was assigned $\nu 7 / 2^{-}[514] \otimes \nu 9 / 2^{+}[624] \otimes \pi 5 / 2^{+}[402] \otimes$ $\pi 7 / 2^{+}[404]$ as the dominant configuration [55]. In Fig. 7, we show the calculated result for this configuration. While the calculation yields excellent agreement with the experimental level at 3.265 MeV [55], we further predict a rotational based on it (sideband 5).

\section{5. ${ }^{182} \mathrm{~W}$}

Apart form the yrast band, several multi-quasiparticle high$K$ bands are experimentally known for the $N=108$ isotope ${ }^{182} \mathrm{~W}$ [56-58]. The yrast band is established up to $I=20 \hbar$. In Fig. 8, we compare our calculated results with experimental data. The suggested predominant quasiparticle structures of the high- $K$ bands are given in Table VIII. The two-qp band with
$K=4^{-}$is known to have qusineutron structure with dominant configuration of $v 1 / 2^{-}[510] \otimes v 9 / 2^{+}$[624]. Interestingly, this band indicates a signature effect in the medium spin region. Our calculations ("sideband 1" in Fig. 8) can reproduce that effect but it is less prominent than the experimental one. The $K=10^{+}$band was experimentally established up to $I=19 \hbar$ and suggested to have two-quasineutron structure. We obtain the predominant configuration as $v 9 / 2^{+}[624] \otimes v 11 / 2^{+}[615]$, which is consistent with the experimental suggestion. Since this band is based on two high- $K i_{13 / 2}$ quasineutron orbitals, signature splitting is not observed for this band. This is a general feature for $t$ bands as discussed by Frauendorf [65].

The two four-qp bands with $K^{\pi}=15^{+}$and $17^{-}$are experimentally known with bandhead energies less than 4.0 MeV. These bands become yrast at $I>16$ [56]. We have qualitatively described these two bands. According to our calculation, the predominant configurations of these bands are $\nu 7 / 2^{-}[503] \otimes \nu 9 / 2^{+}[624] \otimes \pi 5 / 2^{+}[402] \otimes \pi 9 / 2^{-}[514]$ and $\quad v 9 / 2^{+}[624] \otimes v 11 / 2^{+}[615] \otimes \pi 5 / 2^{+}[402] \otimes \pi 9 / 2^{-}$ [514], respectively.

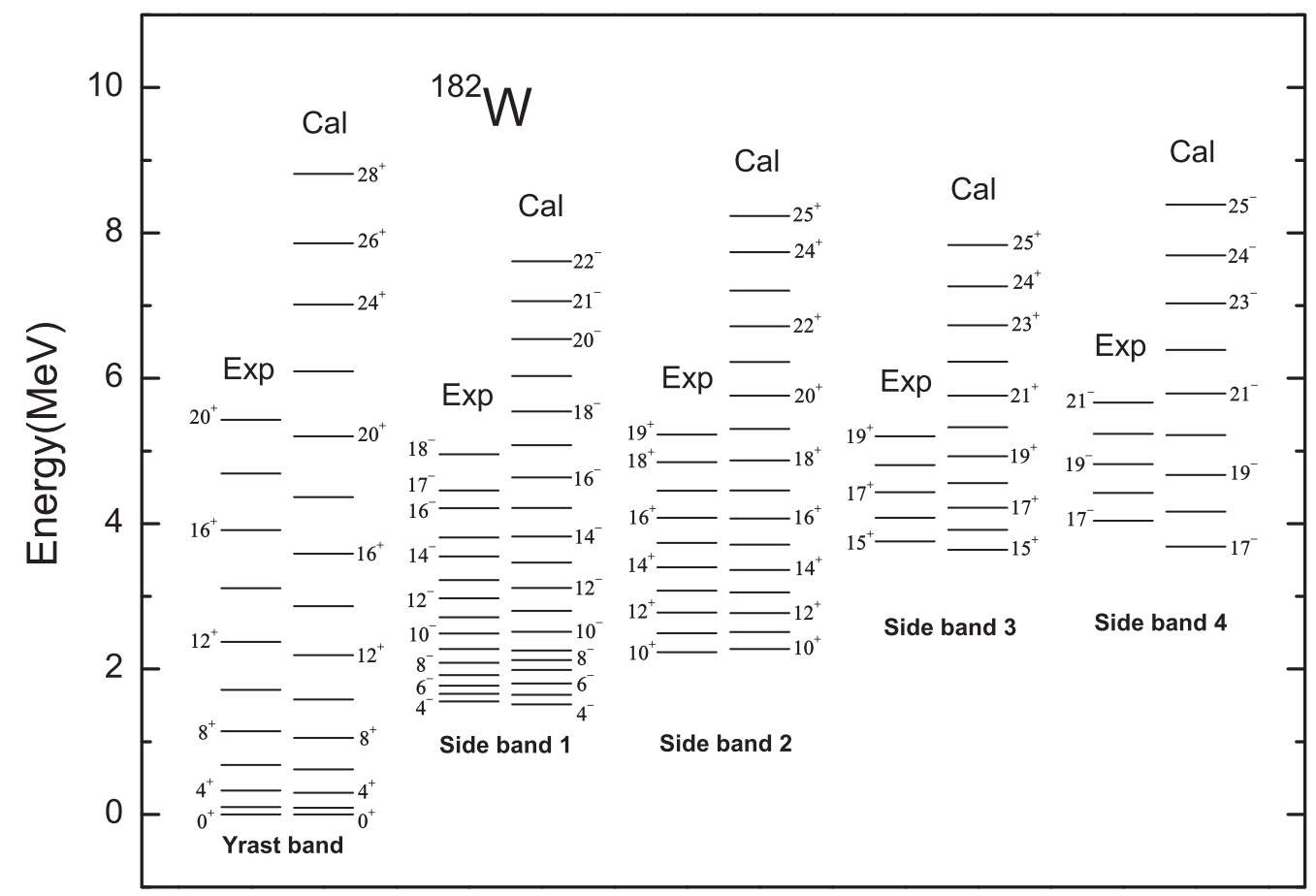

FIG. 8. Comparison of calculated energies with available data for ${ }^{182} \mathrm{~W}$. Experimental values are taken from Refs. [56-58]. 
TABLE VIII. Main configurations of the side bandheads of ${ }^{182} \mathrm{~W}$.

\begin{tabular}{lccc}
\hline \hline Band & Type & $K^{\pi}$ & Configuration \\
\hline Side 1 & Two-qp & $4^{-}$ & $v 1 / 2^{-}[510] \otimes 9 / 2^{+}[624]$ \\
Side 2 & Two-qp & $10^{+}$ & $\nu 9 / 2^{+}[624] \otimes 11 / 2^{+}[615]$ \\
Side 3 & Four-qp & $15^{+}$ & $v 7 / 2^{-}[503] 9 / 2^{+}[624] \otimes \pi 5 / 2^{+}[402] 9 / 2^{-}[514]$ \\
Side 4 & Four-qp & $17^{-}$ & $\nu 9 / 2^{+}[624] 11 / 2^{+}[615] \otimes \pi 5 / 2^{+}[402] 9 / 2^{-}[514]$ \\
\hline \hline
\end{tabular}

\section{6. ${ }^{184} \mathrm{~W}$}

The energy levels for ${ }^{184} \mathrm{~W}$ are depicted in Fig. 9. The experimental yrast band is known up to $16 \hbar$. Our calculation have reproduced the known yrast data very well and predicted the same band up to spin $28 \hbar$ with a good rotational structure. A two-qp $K=5^{-}$state with configuration $v 1 / 2^{-}[510] \otimes$ $v 11 / 2^{+}[615]$ was reported by Wheldon et al. [63]. Comparing with the PSM results, we see that our calculated $I^{\pi}=5^{-}$ state of this configuration lies about $1.0 \mathrm{MeV}$ higher than the experimental energy of $1.285 \mathrm{MeV}$, whereas our calculated $5^{-}$state with the neutron two-qp configuration $v 1 / 2^{-}[510] \otimes$ $v 9 / 2^{+}[624]$ matches well with the experimental energy. This leads us to suggest that the $5^{-}$band has a dominant configuration of $v 1 / 2^{-}[510] \otimes v 9 / 2^{+}[624]$ (compare cal 1 and cal 2 of sideband 1 in Table IX).

A similar situation occurs also for the experimentally known $I^{\pi}=7^{-}$state. Our PSM calculation tends to reassign the configuration of this state as $\pi 5 / 2^{+}[402] \otimes \pi 9 / 2^{-}[514]$ (cal 2 of sideband 2 in Fig. 9). This differs from the previously suggested configuration $v 3 / 2^{-}[512] \otimes v 11 / 2^{+}[615]$ because our calculation for this neutron two-qp configuration lies much higher in energy (cal 1 of sideband 2 in Fig. 9).
Wheldon et al. [63] also observed a $188 \pm 38$-ns isomer at $3862 \mathrm{keV}$ for the first time, but the spin parity as well as the quasiparticle structure of this state have remained uncertain. The PSM calculation suggests one possible four-qp configuration for this level as the $K^{\pi}=$ $18^{-}$one with $v 9 / 2^{+}[624] \otimes v 11 / 2^{+}[615] \otimes \pi 7 / 2^{+}[404] \otimes$ $\pi 9 / 2^{-}[514]$ (see Table IX). In fact, the calculated bandhead level of $I^{\pi}=18^{-}$matches well the experimental $3862-\mathrm{keV}$ level. However, the calculation shows that there is another $I^{\pi}=17^{-}$level also very close to the observed level. This has the four-qp configuration $v 9 / 2^{+}[624] \otimes v 11 / 2^{+}[615] \otimes$ $\pi 5 / 2^{+}[402] \otimes \pi 9 / 2^{-}$[514]. At present, it is difficult to assert which of the four-qp configurations should be assigned to the experimental level. However, our calculation shows a clear difference in MoI between the two possible configurations. From Fig. 9, it is obvious that the band starting from $I^{\pi}=18^{-}$ has smaller energy intervals, corresponding to a larger MoI. Thus further experimental measurement is necessary in order to correctly ascribe its structure.

$$
\text { 7. }{ }^{186} \mathrm{~W}
$$

The experimental information on ${ }^{186} \mathrm{~W}$ is presently rather limited. As shown in Fig. 10, our PSM calculation has well

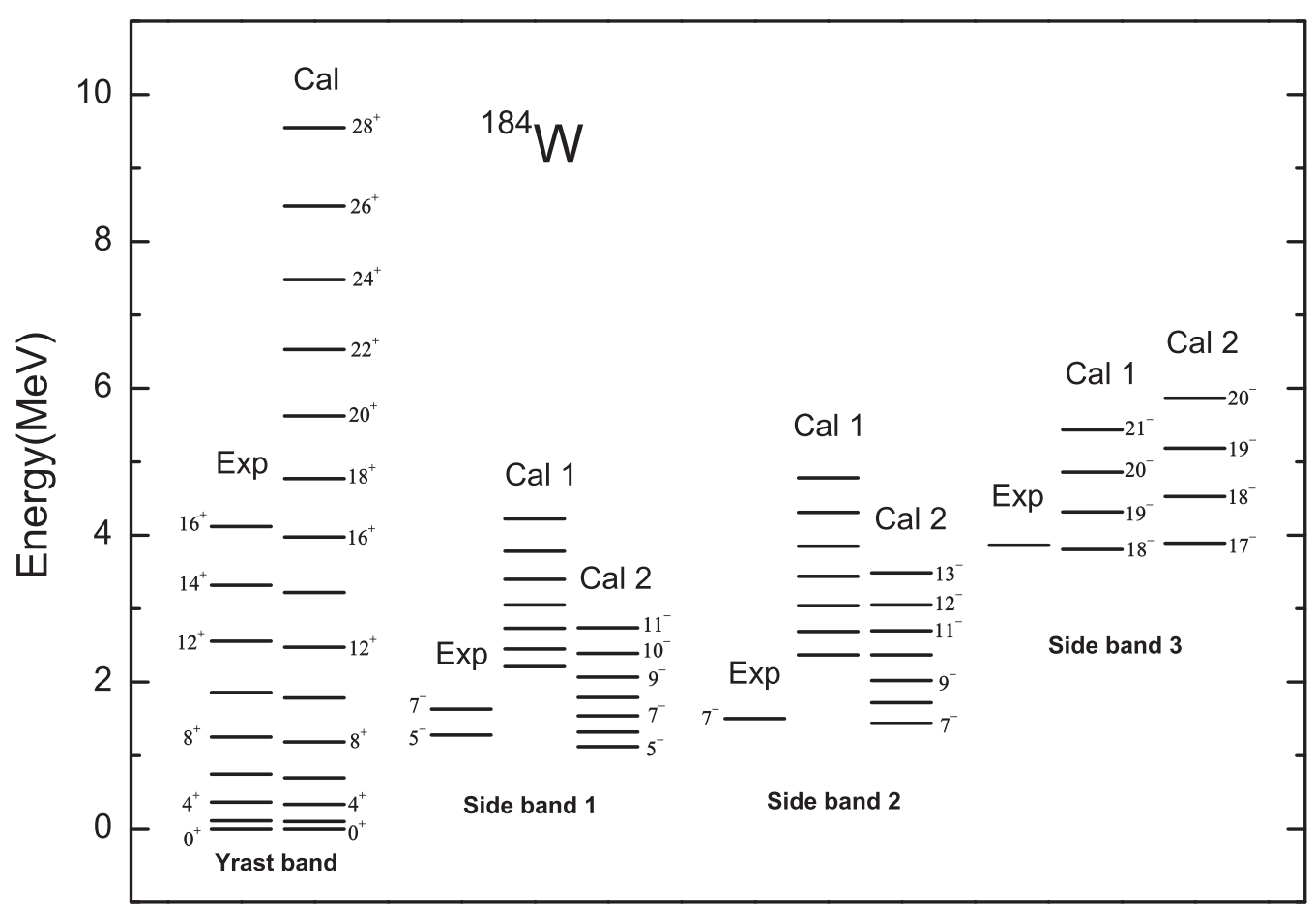

FIG. 9. Comparison of calculated energies with available data for ${ }^{184} \mathrm{~W}$. Experimental values are taken from Refs. [63,64]. 
TABLE IX. Main configurations of the side bandheads of ${ }^{184} \mathrm{~W}$.

\begin{tabular}{lccc}
\hline \hline Band & Type & $K^{\pi}$ & Configuration \\
\hline Side 1 cal 1 & Two-qp & $5^{-}$ & $\nu 1 / 2^{-}[510] \otimes 11 / 2^{+}[615]$ \\
Side 1 cal 2 & Two-qp & $5^{-}$ & $\nu 1 / 2^{-}[510] \otimes 9 / 2^{+}[624]$ \\
Side 2 cal 1 & Two-qp & $7^{-}$ & $\nu 3 / 2^{-}[512] \otimes 11 / 2^{+}[615]$ \\
Side 2 cal 2 & Two-qp & $7^{-}$ & $\pi 5 / 2^{+}[402] \otimes 9 / 2^{-}[514]$ \\
Side 3 cal 1 & Four-qp & $18^{-}$ & $\nu 9 / 2^{+}[624] 11 / 2^{+}[615] \otimes \pi 7 / 2^{+}[404] 9 / 2^{-}[514]$ \\
Side 3 cal 2 & Four-qp & $17^{-}$ & $\nu 9 / 2^{+}[624] 11 / 2^{+}[615] \otimes \pi 5 / 2^{+}[402] 9 / 2^{-}[514]$ \\
\hline \hline
\end{tabular}

reproduced the known yrast data up to $I=14 \hbar$ and predicted the high-spin part. A $K=2^{-}$band is known, although only a few low-lying states were experimentally identified for this band. Our PSM calculation overestimates the bandhead energy by about $350 \mathrm{keV}$. We suggest that this band has a dominant configuration of $v 7 / 2^{-}[503] \otimes v 11 / 2^{+}[615]$ (see Table X). A $K=7^{-}$isomeric state is also known. Our calculations have found a two-quasiproton band with dominant configuration $\pi 5 / 2^{+}[402] \otimes \pi 9 / 2^{-}[514]$, which correctly reproduces the two known levels of $7^{-}$and $8^{-}$. We further predict the highspin part of this band shown in Fig. 10.

In ${ }^{186} \mathrm{~W}$, there is an experimentally known long-lived isomer at $3.543 \mathrm{MeV}$, tentatively assigned as $16^{+}$for spin/parity. We obtain from our calculation a $16^{+}$band at somewhat higher excitation and predict a rotational band based on it (sideband 3 in Fig. 10). We suggest that this band has a configuration $v 7 / 2^{-}[503] 11 / 2^{+}[615] \otimes \pi 5 / 2^{+}[402] 9 / 2^{-}[514]$ (see Table X).

\section{Electromagnetic properties of the yrast and $K$-isomer bands}

The electric quadrupole transition probability carries useful structure information related to shape when the nucleus rotates. While this quantity usually describes collective properties of the system, it is also sensitive to structure changes in the wave function, particularly when band crossings occur. In the $B(E 2)$

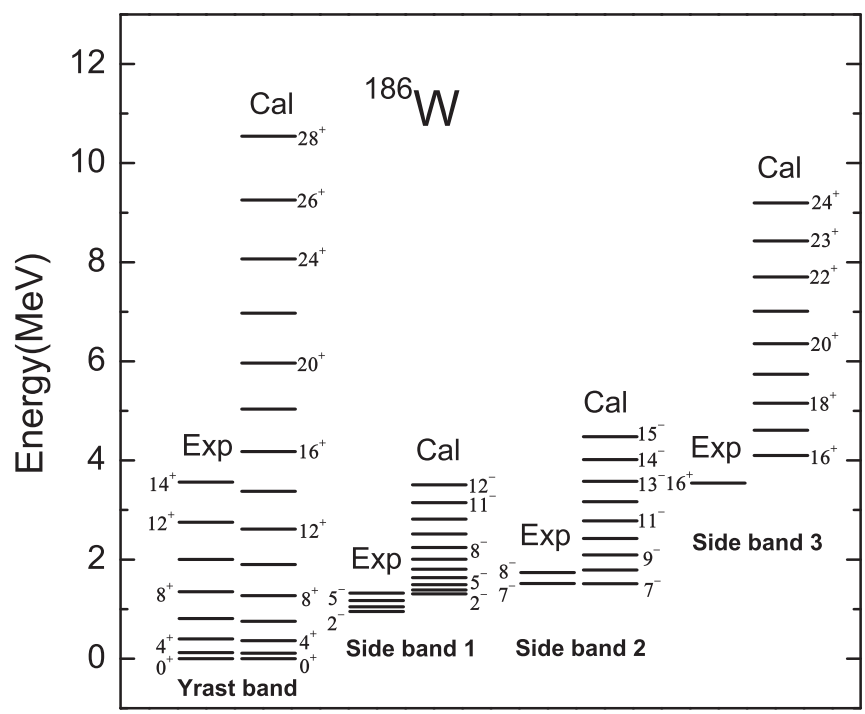

FIG. 10. Comparison of calculated energies with available data for ${ }^{186} \mathrm{~W}$. Experimental values are taken from Refs. [66]. calculations, we have used effective charges $\left(e_{\text {eff }}\right)$ of $1.5 \mathrm{e}$ for protons and $0.5 \mathrm{e}$ for neutrons, which are the standard values used in PSM calculations [21]. On the other hand, the magnetic dipole transition probability $B(M 1)$ and $g$-factor are known to be sensitive probes of any variation of single-particle structures in the wave function. In particular, these quantities distinguish the proton or neutron contributions to the wave function as their orbital part of the operators has contributions only from protons and their spin operators carry opposite signs due to

$$
\begin{aligned}
& g_{l}^{\pi}=1, \quad g_{s}^{\pi}=5.586 \times 0.75, \\
& g_{l}^{\nu}=0, \quad g_{s}^{\nu}=-3.826 \times 0.75 .
\end{aligned}
$$

Here $g_{s}^{\pi}$ and $g_{s}^{v}$ are damped by the usual 0.75 factor from the free-nucleon values to account for the core-polarization and meson-exchange current corrections [67]. The same quenching value is used for all $B(M 1)$ and $g$-factor calculations in the present study. The formulas for $B(E 2), B(M 1)$, and $g$-factor calculation in the PSM framework can be found in the early PSM works, for example, in Refs. [68,69].

\section{B(E2) and g-factor of the yrast bands}

In Fig. 11, the calculated $B(E 2)$ 's for the yrast band of even-even ${ }^{174-186} \mathrm{~W}$ are depicted and compared with available experimental data. The theoretical results indicate an overall trend of the $B(E 2)$ 's as a function of spin, in which one sees a smooth increase at low spins and near constant values at high spins. In the middle spin range, a disturbance occurs for the three lighter isotopes $\left({ }^{174,176,178} \mathrm{~W}\right)$ where a sudden drop in $B(E 2)$ is seen. The available experimental data seem to support this overall behavior, although the theoretical calculation deviates from some variation details seen in the data. In Fig. 11(a), for example, the theoretical $B(E 2)$ values show a later drop in the middle spin range compared to experiment. Nevertheless, this variation can be understood as corresponding to the structural changes in the yrast wave functions because of band crossings as seen in Fig. 2(a). E2 transition probabilities are not measured yet for ${ }^{176} \mathrm{~W}$ and those for ${ }^{178,180} \mathrm{~W}$ are available only for the lowest state transitions. In ${ }^{182,184,186} \mathrm{~W}, B(E 2)$ values are known from experiment up to reasonably high spins. At low spins, a smooth increase in the $B(E 2)$ values is observed, which our calculated results agree with nicely. At higher spins, small staggerings in $B(E 2)$ are seen in data but cannot be precisely reproduced by the present calculation.

The calculated $g$-factors for the yrast bands are illustrated in Fig. 12 for all the $\mathrm{W}$ isotopes studied here. The theoretical results are presented separately for the proton and neutron 
TABLE X. Main configurations of the side bandheads of ${ }^{186} \mathrm{~W}$.

\begin{tabular}{lccc}
\hline \hline Band & Type & $K^{\pi}$ & Configuration \\
\hline Side 1 & Two-qp & $2^{-}$ & $v 7 / 2^{-}[503] \otimes 11 / 2^{+}[615]$ \\
Side 2 & Two-qp & $7^{-}$ & $\pi 5 / 2^{+}[402] \otimes 9 / 2^{-}[514]$ \\
Side 3 & Four-qp & $16^{+}$ & $v 7 / 2^{-}[503] 11 / 2^{+}[615] \otimes \pi 5 / 2^{+}[402] 9 / 2^{-}[514]$ \\
\hline \hline
\end{tabular}

contributions, and the sums of them (illustrated in Fig. 12 as "Yrast total") are to be compared with the data. As discussed earlier, structural changes near band crossings along the yrast line are clearly demonstrated here through the variation of $g$-factors. This can be seen in the spin range $I=12-18 \hbar$ as a general decreasing trend of the total $g$-factors due to the enhanced neutron single-particle components in the wave functions. The increasing trend of $g$-factors after $I=18 \hbar$ is
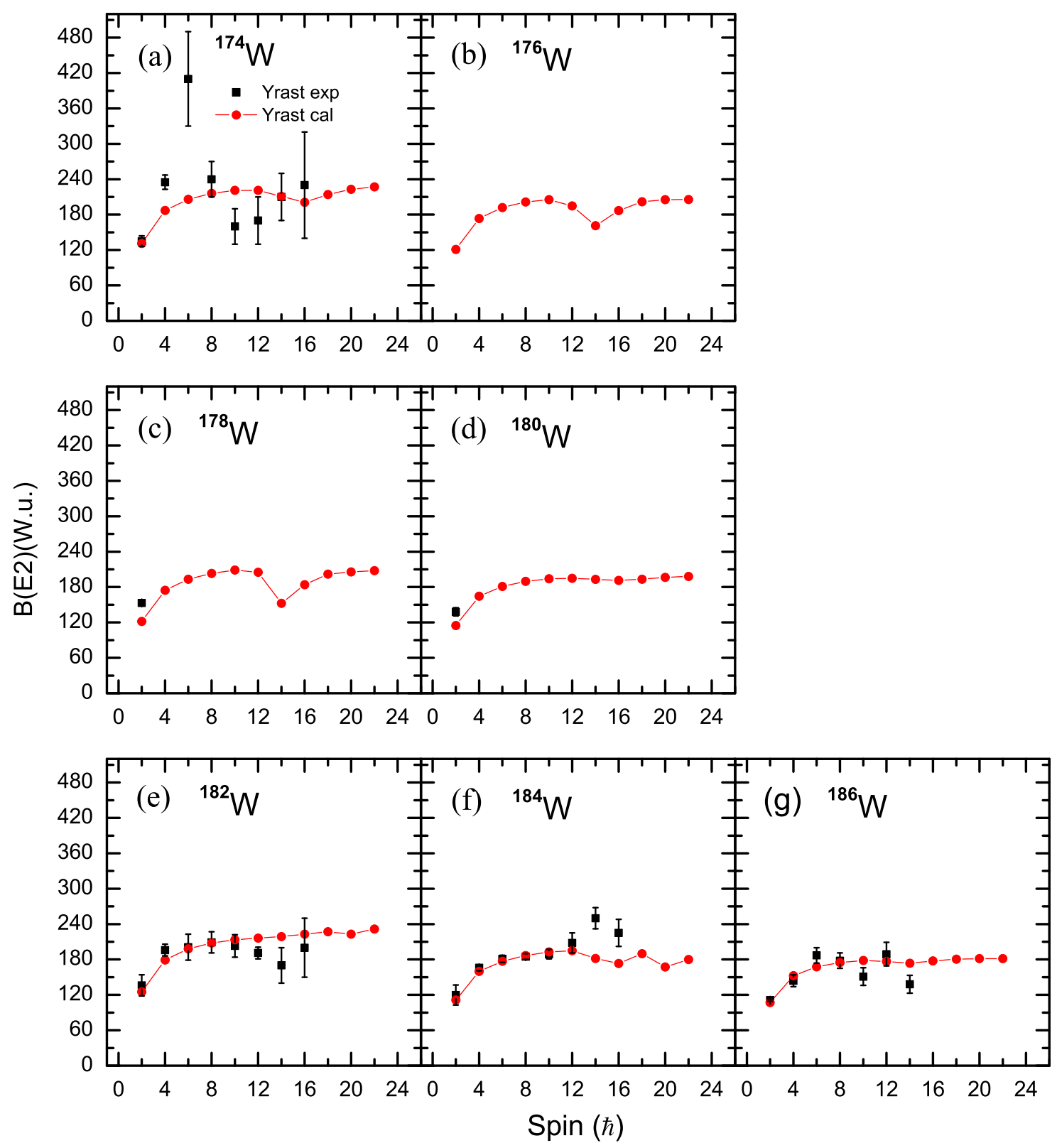

FIG. 11. Comparison of calculated $B(E 2)$ values (in W.u.) with available data for the yrast band of (a) ${ }^{174} \mathrm{~W}$ (data taken from Ref. [45]), (b) ${ }^{176} \mathrm{~W}$, (c) ${ }^{178} \mathrm{~W}$ (data taken from Ref. [50]), (d) ${ }^{180} \mathrm{~W}$ (data taken from Ref. [55]), (e) ${ }^{182} \mathrm{~W}$ (data taken from Ref. [58]), (f) ${ }^{184} \mathrm{~W}$ (data taken from Ref. [64]), and (g) ${ }^{186} \mathrm{~W}$ (data taken from Ref. [66]). 

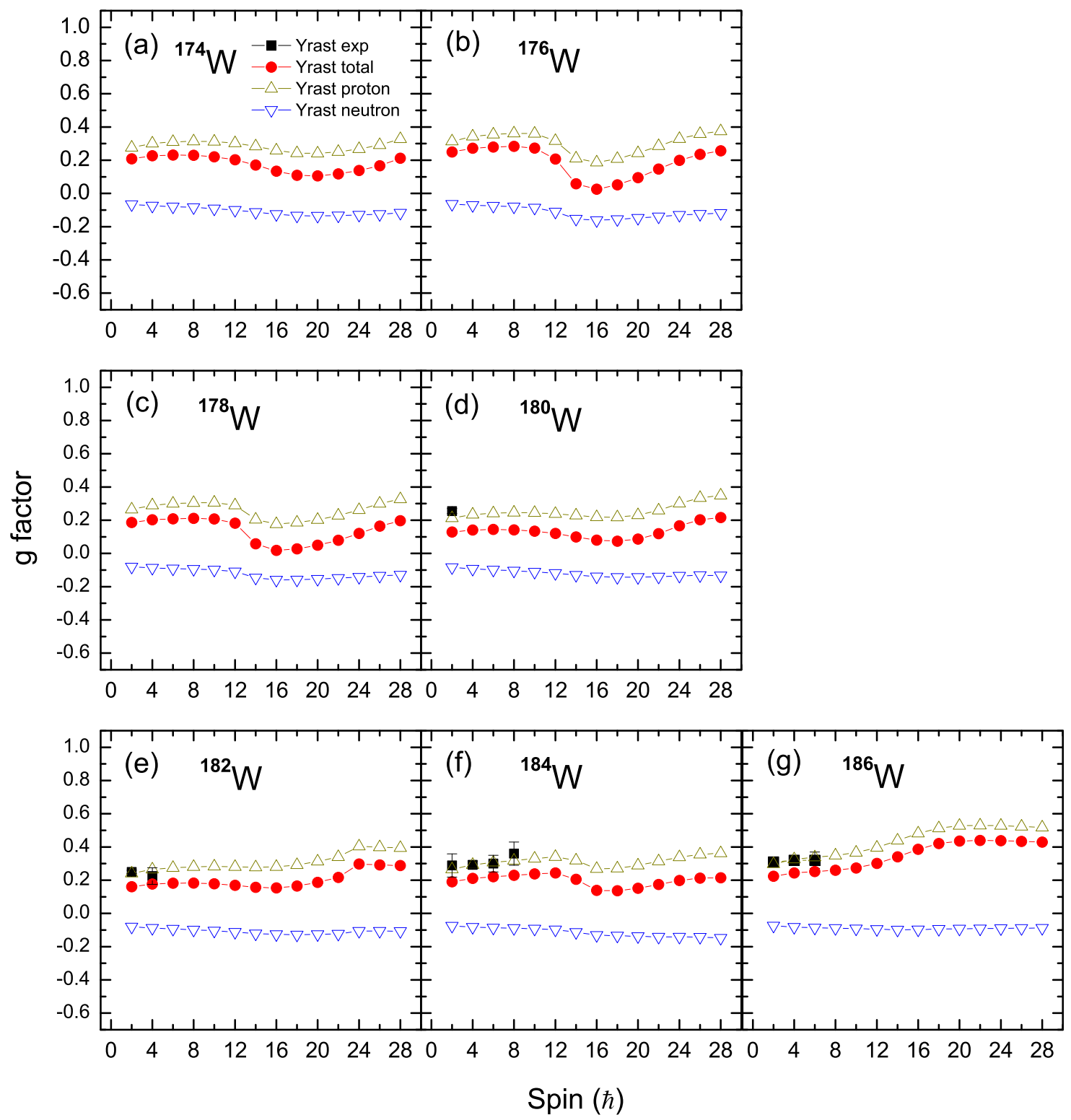

FIG. 12. Comparison of calculated $g$-factor values with available data for the yrast band of (a) ${ }^{174} \mathrm{~W}$, (b) ${ }^{176} \mathrm{~W}$, (c) ${ }^{178} \mathrm{~W}$, (d) ${ }^{180} \mathrm{~W}$, (e) ${ }^{182} \mathrm{~W}$, (f) ${ }^{184} \mathrm{~W}$, and $(\mathrm{g}){ }^{186} \mathrm{~W}$. Experimental values are taken from Ref. [72].

attributed to the contribution of the proton single particles in the wave functions because after that spin, four-qp and higherqp states with one or more proton pairs in the configurations are the major components (see Table III). The experimental values are not known for the lighter-mass nuclei $\left({ }^{174,176,178} \mathrm{~W}\right)$. The comparison with the known $g$-factor data indicates that the calculated results (red dots) slightly underestimate the data. The same problem was found earlier in Ref. [70]. Modifications in the damping factor in Eq. (10) can certainly improve the agreement but we prefer to keep using the standard values.

\section{B(E2), B(M1), and $g$-factor of the K-isomer bands}

In Ref. [46], Crowell et al. presented the experimental $B(M 1) / B(E 2)$ ratios for the four-qp $14^{+}$band in ${ }^{176} \mathrm{~W}$ (sideband 1 in Fig. 5). This is a valuable piece of information to be used to test theoretical models because the configuration includes four single nucleons residing in four high- $K$ orbitals (see Table V). On the basis that our PSM calculation already obtained good results for the energy levels of this nucleus (Fig. 5), we calculate the $B(M 1) / B(E 2)$ ratios for this four-qp $14^{+}$band, with the results compared with the data in Fig. 13. The calculation can be said to be satisfactory because the overall variation trend is reproduced correctly although the theoretical values are 2-3 times too large when compared to the data. This discrepancy comes mainly from the $B(M 1)$ calculation as the $B(E 2)$ calculation can usually yield correct results, as already seen in Fig. 11.

There has been accumulated $B(E 2)$ and $B(M 1)$ data from decays of the isomeric states in this mass region. The understanding of these data is important for the study of the 


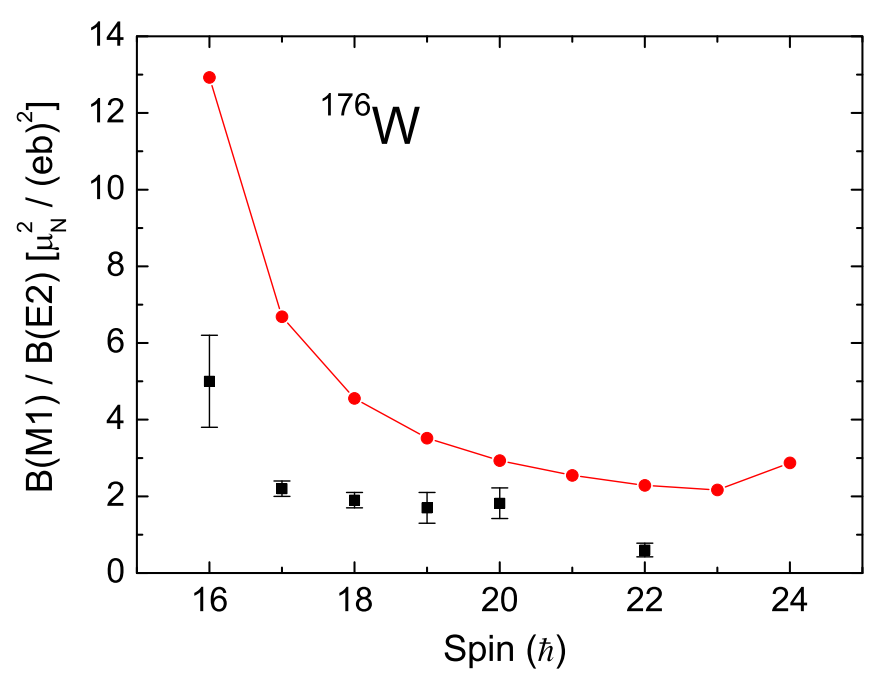

FIG. 13. Comparison of calculated $B(M 1) / B(E 2)$ ratios of sideband 1 in Fig. 5 with available data for ${ }^{176} \mathrm{~W}$. Experimental values are taken from Ref. [46].

$K$-selection rule and its violation. A systematic description of these data is a theoretical challenge. In Table XI, we present our calculations for those isomer decays where data exist and compare our theoretical results with the available experimental data. The calculation includes the allowed transitions that involve the initial and final states with similar $K$ quantum numbers and also those $K$-forbidden transitions for which the

TABLE XI. Comparison of calculated $B(E 2)$ and $B(M 1)$ values (in W.u.) for the isomeric states in $\mathrm{W}$ with the available data taken from Refs. [50,55,58,64].

\begin{tabular}{lccclc}
\hline \hline$K_{i}^{\pi}$ & $I_{i}$ & $K_{f}^{\pi}$ & $I_{f}$ & \multicolumn{2}{c}{$B\left(E 2, M 1 ; I_{i} \rightarrow I_{f}\right)$} \\
\cline { 5 - 6 } & & & & & Expt. \\
\hline${ }^{178} \mathrm{~W}$ & & & & & \\
$6^{+}$ & 6 & $0^{+}$ & 4 & $B(E 2)=4.67 \times 10^{-4}$ & $4.56 \times 10^{-8}$ \\
$14^{-}$ & 14 & $13^{-}$ & 13 & $B(M 1)=6.7 \times 10^{-3}$ & $1.25 \times 10^{-5}$ \\
$21^{-}$ & 21 & $18^{-}$ & 20 & $B(M 1)=3.9 \times 10^{-4}$ & $5.36 \times 10^{-7}$ \\
${ }^{180} \mathrm{~W}$ & & & & & \\
$14^{-}$ & 14 & $8^{-}$ & 12 & $B(E 2)=2.8 \times 10^{-6}$ & $2.11 \times 10^{-7}$ \\
$182 \mathrm{~W}$ & & & & & \\
$4^{-}$ & 4 & $2^{-}$ & 2 & $B(E 2)=0.7$ & $9.03 \times 10^{-3}$ \\
$4^{-}$ & 4 & $2^{-}$ & 3 & $B(E 2)=2.6$ & $1.09 \times 10^{-2}$ \\
$4^{-}$ & 4 & $2^{-}$ & 3 & $B(M 1)=1.19 \times 10^{-4}$ & $9.06 \times 10^{-5}$ \\
$4^{-}$ & 4 & $2^{-}$ & 4 & $B(E 2)=5.2$ & $1.92 \times 10^{-2}$ \\
$4^{-}$ & 4 & $2^{-}$ & 4 & $B(M 1)=6.24 \times 10^{-3}$ & $7.24 \times 10^{-4}$ \\
$10^{+}$ & 10 & $0^{+}$ & 10 & $B(M 1)=7.0 \times 10^{-8}$ & $1.19 \times 10^{-8}$ \\
$10^{+}$ & 10 & $0^{+}$ & 8 & $B(E 2)=1.9 \times 10^{-6}$ & $2.84 \times 10^{-7}$ \\
$15^{+}$ & 15 & $10^{+}$ & 13 & $B(E 2)=5.3 \times 10^{-4}$ & $9.14 \times 10^{-7}$ \\
$15^{+}$ & 15 & $10^{+}$ & 14 & $B(M 1)=1.3 \times 10^{-7}$ & $1.07 \times 10^{-9}$ \\
$184 \mathrm{~W}$ & & & & & \\
$5^{-}$ & 5 & $2^{-}$ & 3 & $B(E 2)=0.019$ & $4.41 \times 10^{-3}$ \\
$7^{-}$ & 7 & $6^{-}$ & 6 & $B(E 2)=1.8$ & $8.52 \times 10^{-1}$ \\
$7^{-}$ & 7 & $6^{-}$ & 6 & $B(M 1)=5.2 \times 10^{-3}$ & $3.87 \times 10^{-3}$ \\
$7^{-}$ & 7 & $5^{-}$ & 5 & $B(E 2)=3.09$ & $1.17 \times 10^{-1}$ \\
\hline \hline & & & & &
\end{tabular}

initial and final states differ very much in $K$, by up to 10 units of $\hbar$, see Table XI.

Comparing our theoretical values with data, one sees that, for most of the transitions, our model works reasonably well with the deviation of the results from data within an order of magnitude. However, there are cases where the theoretical and experimental values differ by several orders of magnitude, for example, the $6^{+}$isomer $B(E 2)$ and the $21^{-}$isomer $B(M 1)$ transitions in ${ }^{178} \mathrm{~W}$ and the $15^{+}$isomer $B(E 2)$ transitions in ${ }^{182} \mathrm{~W}$. In all the cases where large deviations occur, we find that the calculated transition rates are always too small as compared to data. This reveals that $K$ mixing in the model may not be strong enough to yield sufficiently large isomer decay rates. Specific effects beyond the present model may need to be considered. In Ref. [71], it was demonstrated that the $K$-forbidden transition from the $6^{+}$two-qp isomer to the ground-state band is sensitive to mixing with the $6^{+}$ state of the $\gamma$-vibrational band, which could be accounted for when a triaxial deformed single-particle basis with threedimensional angular-momentum projection is employed in the model.

Finally, it is interesting to discuss $g$-factors of the isomeric states, which are unique quantities to reflect single-particle compositions in multi-qp high- $K$ configurations. In Table XII, we summarize the calculated $g$-factors of all the $K$-isomeric states that have been studied in the previous sections. For these isomers, we know their lifetime experimentally but not their structure. We list the proposed configurations and give comparison of the bandhead energies of these $K$ isomers with their known experimental values. Most of the $g$-factors are our predictions which can be useful for future experiments to look for. It is very much desired to see if some of the predicted isomeric $g$-factors can be measured.

In Table XII, one sees some $g$-factors having a very large value more than 1 . Typically, these belong to those proton two-qp states such as the $5^{-}$isomer in ${ }^{180} \mathrm{~W}$ and the $7^{-}$isomer in ${ }^{184,186} \mathrm{~W}$. On the other hand, the neutron two-qp states typically have very small or even negative $g$-factors. Examples of such are the $6^{+}$and $7^{-}$isomers in ${ }^{178} \mathrm{~W}$, the $4^{-}$and $10^{+}$ isomers in ${ }^{182} \mathrm{~W}$, and the two $5^{-}$and one $7^{-}$isomers in ${ }^{184} \mathrm{~W}$. For those four- or higher-qp states which are combinations of proton and neutron single particles, their $g$-factors normally have a value between the above two extremes and depend very much on their structure. For the only existing data of the four-qp $14^{+}$isomer in ${ }^{176} \mathrm{~W}$, our calculated $g$-factor of 0.58 compares reasonably well with the measured one [0.48(2)]. For the eight-qp, $25^{+}$isomer in ${ }^{178} \mathrm{~W}$, we predict its $g$-factor to be 0.43 , a moderate value presumably coming from the combined contribution of the four individual proton and the four individual neutron orbitals.

For several of the observed high- $K$ structures, $g$-factors could also be obtained from in-band $\gamma$-ray branching ratios, albeit requiring additional assumptions. A systematic analysis of this kind will be the subject of future work.

\section{SUMMARY AND CONCLUSIONS}

Modern experimental facilities with large $\gamma$-ray detector arrays with unprecedented sensitivity and resolution provide 
TABLE XII. Bandhead energy (BHE) and $g$-factor of $K$-isomeric configurations.

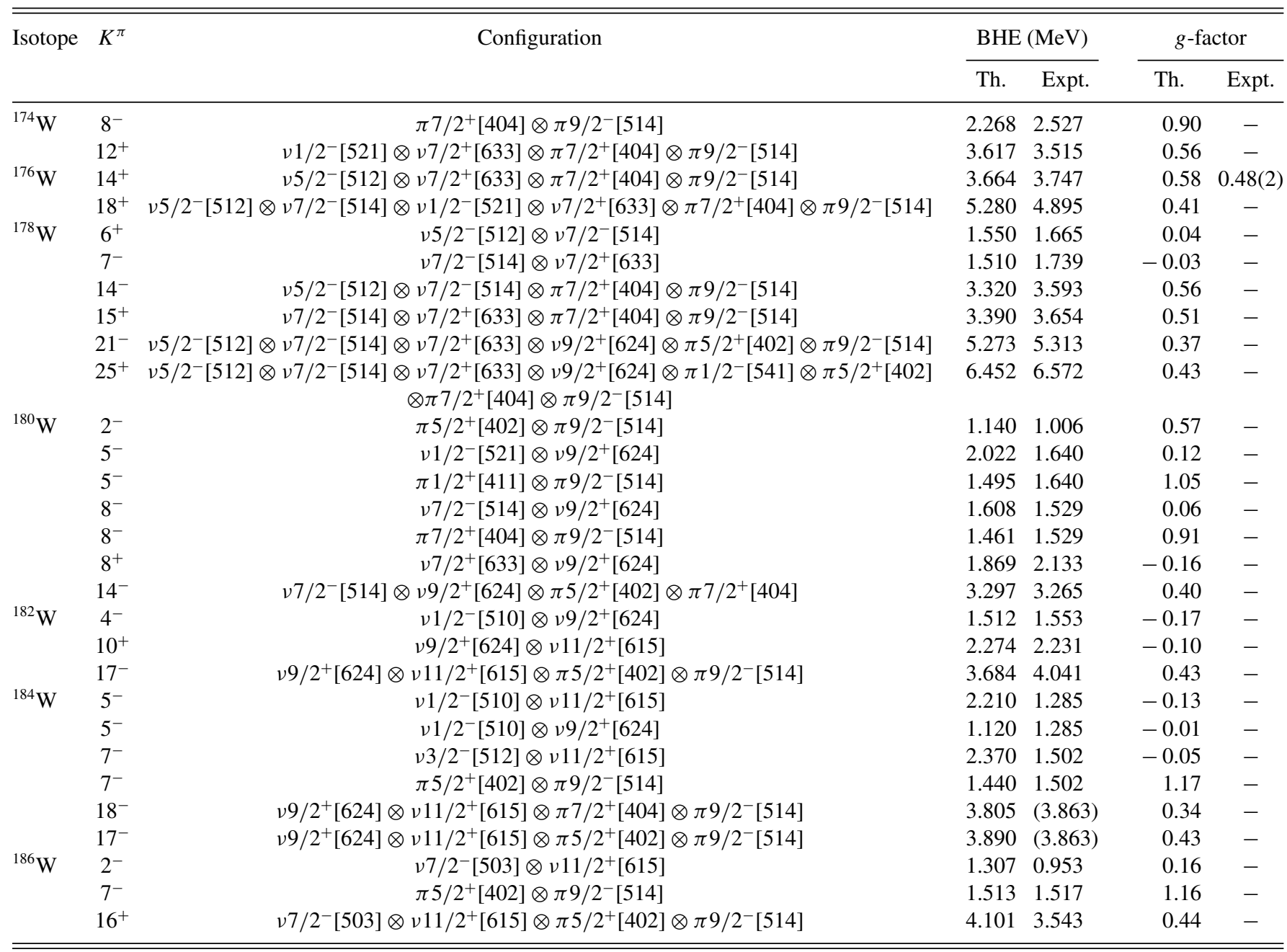

fascinating high-spin data. This stream of new data reveals many interesting observations regarding nuclear excitation modes. New findings present a challenge to nuclear theory for a better understanding of the nuclear many-body problem. It has been emphasized that the multi-quasiparticle high- $K$ excitations must be systematically tested against the nuclear models. With this view in mind, the PSM configuration space has been recently extended by the Pfaffian method to include up to 10-qp configurations in calculations.

In the present paper, we have systematically analyzed the structure of the high-spin regime as well as multi-qp high- $K$ isomers of even-even ${ }^{174-186} \mathrm{~W}$ isotopes. The structure of yrast high-spin levels is analyzed for multi-quasiparticle excitations. Apart from the yrast band, $K$ isomers and rotational bands based on those are described and their dominant multi-quasiparticle structures are predicted. The electromagnetic properties [e.g., $B(E 2), B(M 1), g$-factor] of the $K$ isomers are also studied and compared with available experimental data. For most of the $K$ isomers, the experimental properties are unknown and our results remain as predictions. With the development of modern experimental facilities, in particular radioactive ion beam accelerators and $\gamma$-ray tracking arrays, the extreme states in exotic nuclei are becoming more accessible.

\section{ACKNOWLEDGMENTS}

Research at Shanghai Jiao Tong University is supported by the National Natural Science Foundation of China (Grant No. 11575112), by the National Key Program for S\&T Research and Development (Grant No. 2016YFA0400501), and by the 973 Program of China (Grant No. 2013CB834401). This work is also partially supported by the UK Science and Technology Facilities Council under Grant No. ST/L005743/1.
[1] P. Walker and G. Dracoulis, Nature (London) 399, 35 (1999).

[2] P. M. Walker and F. R. Xu, Phys. Scr. 91, 013010 (2016).
[3] G. D. Dracoulis, P. M. Walker, and F. G. Kondev, Rep. Prog. Phys. 79, 076301 (2016). 
[4] P. M. Walker, J. Phys. G: Nucl. Phys. 34, 123 (2007).

[5] P. M. Walker and J. J. Carroll, Phys. Today 58(6), 39 (2005).

[6] F. R. Xu, E. G. Zhao, R. Wyss, and P. M. Walker, Phys. Rev. Lett. 92, 252501 (2004).

[7] R.-D. Herzberg, P. T. Greenlees, P. A. Butler, G. D. Jones et al., Nature (London) 442, 896 (2006).

[8] C. S. Purry, P. M. Walker, G. D. Dracoulis, T. Kibédi et al., Phys. Rev. Lett. 75, 406 (1995).

[9] G. D. Dracoulis, G. J. Lane, F. G. Kondev, A. P. Byrne et al., Phys. Rev. C 71, 044326 (2005).

[10] M. W. Reed, I. J. Cullen, P. M. Walker, Y. A. Litvinov et al., Phys. Rev. Lett. 105, 172501 (2010).

[11] A. Aprahamian and Y. Sun, Nat. Phys. 1, 81 (2005).

[12] Y. Sun, M. Wiescher, A. Aprahamian, and J. Fisker, Nucl. Phys. A 758, 765 (2005).

[13] D. Belic, C. Arlandini, J. Besserer, J. de Boer et al., Phys. Rev. Lett. 83, 5242 (1999).

[14] P. M. Walker, G. D. Dracoulis, and J. J. Carroll, Phys. Rev. C 64, 061302 (2001).

[15] S. Frauendorf, K. Neergård, J. A. Sheikh, and P. M. Walker, Phys. Rev. C 61, 064324 (2000).

[16] S. Frauendorf, Nucl. Phys. A 677, 115 (2000).

[17] D. Almehed, S. Frauendorf, and F. Dönau, Phys. Rev. C 63, 044311 (2001).

[18] S.-I. Ohtsubo and Y. R. Shimizu, Nucl. Phys. A 714, 44 (2003).

[19] Y. R. Shimizu, M. Matsuzaki, and K. Matsuyanagi, Phys. Rev. C 72, 014306 (2005).

[20] F. R. Xu, P. M. Walker, and R. Wyss, Phys. Rev. C 62, 014301 (2000).

[21] K. Hara and Y. Sun, Int. J. Mod. Phys. E 04, 637 (1995).

[22] Y. Sun, Phys. Scr. 91, 043005 (2016).

[23] L.-J. Wang, F.-Q. Chen, T. Mizusaki, M. Oi, and Y. Sun, Phys. Rev. C 90, 011303 (2014).

[24] L.-J. Wang, Y. Sun, T. Mizusaki, M. Oi, and S. K. Ghorui, Phys. Rev. C 93, 034322 (2016).

[25] J. Jolie and A. Linnemann, Phys. Rev. C 68, 031301 (2003).

[26] R. B. Firestone, C. M. Baglin, and S. Y. F. Chu, Table of Isotopes, Vol. 4 (Wiley, New York, 1999).

[27] P. Ring and P. Schuck, The Nuclear Many-Body Problem (Springer-Verlag, New York, 1980).

[28] Y. Sun and K. Hara, Comput. Phys. Commun. 104, 245 (1997).

[29] T. Mizusaki, M. Oi, F.-Q. Chen, and Y. Sun, Phys. Lett. B 725, 175 (2013)

[30] L. M. Robledo, Phys. Rev. C 79, 021302 (2009).

[31] G. F. Bertsch and L. M. Robledo, Phys. Rev. Lett. 108, 042505 (2012).

[32] M. Oi and T. Mizusaki, Phys. Lett. B 707, 305 (2012).

[33] T. Mizusaki and M. Oi, Phys. Lett. B 715, 219 (2012).

[34] B. Avez and M. Bender, Phys. Rev. C 85, 034325 (2012).

[35] Q.-L. Hu, Z.-C. Gao, and Y. S. Chen, Phys. Lett. B 734, 162 (2014).

[36] T. Bengtsson and I. Ragnarsson, Nucl. Phys. A 436, 14 (1985).

[37] K. Hara and Y. Sun, Nucl. Phys. A 529, 445 (1991).

[38] P. Moller, J. Nix, W. Myers, and W. Swiatecki, At. Data Nucl. Data Tables 59, 185 (1995).

[39] F.-Q. Chen and J. L. Egido, Phys. Rev. C 93, 064313 (2016); 95, 024307 (2017).
[40] M. Wang, G. Audi, F. G. Kondev, W. J. Huang, S. Naimi, and X. Xu, Chin. Phys. C 41, 030003 (2017).

[41] F. Stephens and R. Simon, Nucl. Phys. A 183, 257 (1972).

[42] S. K. Tandel, P. Chowdhury, E. H. Seabury, I. Ahmad et al., Phys. Rev. C 73, 044306 (2006).

[43] V. Vandone, S. Leoni, G. Benzoni, N. Blasi et al., Phys. Rev. C 88, 034312 (2013).

[44] S. K. Tandel, A. J. Knox, C. Parnell-Lampen, U. S. Tandel et al., Phys. Rev. C 77, 024313 (2008).

[45] E. Browne and H. Junde, Nucl. Data Sheets 87, 15 (1999).

[46] B. Crowell, P. Chowdhury, D. J. Blumenthal, S. J. Freeman et al., Phys. Rev. C 53, 1173 (1996).

[47] M. Basunia, Nucl. Data Sheets 107, 791 (2006).

[48] P. M. Walker, G. Sletten, N. L. Gjrup, M. A. Bentley et al., Phys. Rev. Lett. 65, 416 (1990).

[49] C. S. Purry, P. M. Walker, G. D. Dracoulis, T. Kibédi et al., Nucl. Phys. A 632, 229 (1998).

[50] E. Achterberg, O. Capurro, and G. Marti, Nucl. Data Sheets 110, 1473 (2009).

[51] T. Nakatsukasa, K. Matsuyanagi, M. Matsuzaki, and Y. R. Shimizu, Phys. Scr. 91, 073008 (2016).

[52] P.-A. Söderström et al., Phys. Rev. C 81, 034310 (2010); E. Ideguchi et al., ibid. 94, 064322 (2016); P.-A. Söderström et al., Phys. Lett. B 762, 404 (2016).

[53] S. K. Ghorui, B. B. Sahu, C. R. Praharaj, and S. K. Patra, Phys. Rev. C 85, 064327 (2012).

[54] Z. Patel, P.-A. Söderström, Z. Podolyák, P. H. Regan et al., Phys. Rev. Lett. 113, 262502 (2014).

[55] S.-C. Wu and H. Niu, Nucl. Data Sheets 100, 483 (2003).

[56] P. H. Regan, P. M. Walker, G. D. Dracoulis, S. S. Anderssen et al., Nucl. Phys. A 567, 414 (1994).

[57] T. Shizuma, S. Mitarai, G. Sletten, R. A. Bark et al., Nucl. Phys. A 593, 247 (1995).

[58] B. Singh and J. C. Roediger, Nucl. Data Sheets 111, 2081 (2010).

[59] L. G. Mann, J. B. Carlson, R. G. Lanier, G. L. Struble, W. M. Buckley, D. W. Heikkenen, I. D. Proctor, and R. K. Sheline, Phys. Rev. C 19, 1191 (1979).

[60] M. B. Smith, P. M. Walker, G. C. Ball, J. J. Carroll, P. E. Garrett, G. Hackman, R. Propri, F. Sarazin, and H. C. Scraggs, Phys. Rev. C 68, 031302(R) (2003).

[61] P. M. Walker et al., Phys. Lett. B 309, 17 (1993).

[62] J. A. Sheikh, Y. Sun, and P. M. Walker, Phys. Rev. C 57, R26(R) (1998).

[63] C. Wheldon, J. J. Valiente-Dobón, P. H. Regan, C. J. Pearson et al., Eur. Phys. J. A 20, 365 (2004).

[64] C. M. Baglin, Nucl. Data Sheets 111, 275 (2010).

[65] S. Frauendorf, Nucl. Phys. A 557, 259 (1993).

[66] C. M. Baglin, Nucl. Data Sheets 99, 1 (2003).

[67] B. Castel and I. Towner, Modern Theories of Nuclear Moments, Oxford Studies in Nuclear Physics (Clarendon Press, London, 1990).

[68] Y. Sun and J. L. Egido, Nucl. Phys. A 580, 1 (1994).

[69] Y. Sun and J. L. Egido, Phys. Rev. C 50, 1893 (1994).

[70] B.-A. Bian, Y.-M. Di, G.-L. Long, Y. Sun, J.-y. Zhang, and J. A. Sheikh, Phys. Rev. C 75, 014312 (2007).

[71] F.-Q. Chen, Y. Sun, P. M. Walker, G. D. Dracoulis, Y. R. Shimizu, and J. A. Sheikh, J. Phys. G 40, 015101 (2013).

[72] N. J. Stone, At. Data Nucl. Data Tables 90, 75 (2005). 